\title{
Logical Geometries and Information in the Square of Oppositions
}

\author{
Hans Smessaert · Lorenz Demey
}

Received: date / Accepted: date

\begin{abstract}
The Aristotelian square of oppositions is a well-known diagram in logic and linguistics. In recent years, several extensions of the square have been discovered. However, these extensions have failed to become as widely known as the square. In this paper we argue that there is indeed a fundamental difference between the square and its extensions, viz., a difference in informativity. To do this, we distinguish between concrete Aristotelian diagrams (such as the square) and, on a more abstract level, the Aristotelian geometry (a set of logical relations). We then introduce two new logical geometries (and their corresponding diagrams), and develop a formal, well-motivated account of their informativity. This enables us to show that the square is strictly more informative than many of the more complex diagrams.
\end{abstract}

Keywords Square of Oppositions · Logical Geometry · Logical Diagram • Opposition · Implication · Information as Range · Unconnectedness

\section{Introduction}

The Aristotelian square of oppositions is a diagram that displays four formulas, and certain logical relations holding between them. Although traditionally, it was closely

\author{
Hans Smessaert \\ Department of Linguistics \\ KU Leuven \\ Blijde Inkomststraat 21 - bus 3380 \\ BE 3000 Leuven \\ Belgium \\ E-mail: Hans.Smessaert@arts.kuleuven.be \\ Lorenz Demey \\ Center for Logic and Analytic Philosophy \\ KU Leuven \\ Andreas Vesaliusstraat 2 - bus 3220 \\ BE 3000 Leuven \\ Belgium \\ E-mail: Lorenz.Demey@hiw.kuleuven.be
}


associated with Aristotelian syllogistics, it can be used to study many other logical systems, and nowadays also has applications in linguistics. In recent years, many, increasingly complex extensions of the square have been discovered and intensively studied. At first sight, there does not seem to be a fundamental difference between the Aristotelian square and its extensions. In practice, however, there is a major difference in 'popularity': while the square is nearly universally known among logicians and formal linguists, many of the larger diagrams are only known by a few specialists.

The main aim of this paper is to argue that there is indeed a fundamental difference between the square and its extensions, viz., a difference in informativity. To do this, we will develop a formal, well-motivated account of information in (Aristotelian and other) diagrams, and then use it to show that the square is strictly more informative than many of the more complex diagrams.

Our argumentation consists of four main steps. The first step is to distinguish between concrete Aristotelian diagrams (such as the square and its extensions) and, on a more abstract level, the Aristotelian geometry (the set of logical relations visualized in Aristotelian diagrams). This distinction will enable us to provide a more fine-grained analysis later on (in the fourth step).

Second, we will define two new logical geometries, viz., the opposition and implication geometries (and the corresponding types of diagrams). The Aristotelian geometry can advantageously be seen as hybrid between these two new geometries: they solve some problems that have traditionally been associated with the Aristotelian geometry, and they also have several independent motivations.

The third step concerns information in the opposition and implication geometries. We will adopt an account of information that is well-known in logic and semantics, viz., information as range, and show that it can be used to compare the informativity not only of statements (as is usually done), but also of logical relations. This yields an informativity ordering on the opposition and implication geometries. We will show that this ordering is highly intuitive, and also fits well with the structural properties of these geometries.

The fourth and final step brings everything together. We will argue that the Aristotelian square is highly informative in two successive steps. First, we will show that the Aristotelian geometry is informationally optimal: it is hybrid between the opposition and implication geometries not in some random manner, but exactly so as to maximize informativity. Second, within the Aristotelian geometry, we will make a further distinction between more and less informative diagrams, based on whether or not they contain pairs of formulas that are unconnected (i.e., that stand in the least informative opposition and implication relations). It turns out that such minimally informative pairs do not occur in the classical square, but do occur in some of its extensions.

This four-step argumentation is reflected in the structure of the paper. Section 2 provides some historical background and examples of the Aristotelian square and its extensions; most importantly, introduces the geometry/diagram distinction. Section 3 introduces the opposition and implication geometries and discusses their various properties and motivations. Section 4 applies the 'information as range'-perspective to the opposition and implication geometries, and discusses some advantages of this application. Section 5 shows that the Aristotelian geometry is hybrid between the 
opposition and implication geometries in an informationally optimal way; it also introduces the notion of unconnectedness and studies in which Aristotelian diagrams it occurs. Finally, Section 6 wraps things up and suggests some questions for further research. ${ }^{1}$

\section{The Aristotelian Square of Oppositions}

This section introduces the Aristotelian square of oppositions. Subsection 2.1 defines the Aristotelian geometry and its diagrams, and provides some examples of the square in various logical systems, while Subsection 2.2 discusses some extensions to larger diagrams, such as hexagons and octagons. Building on this concise overview, Subsection 2.3 raises the main issue that will be addressed in this paper.

\subsection{A Brief History of the Aristotelian Square}

The Aristotelian square of oppositions has a rich tradition, originating-together with the discipline of logic itself-in Aristotle's logical works. It has been used and described by some of the most distinguished scholars in the history of philosophical logic, such as Avicenna (Chatti 2012), Anselm of Canterbury (Uckelman 2009), John Buridan (Hughes 1987; Read 2012b), Boole and Frege (Peckhaus 2012). ${ }^{2}$ Contemporary logicians too have found it worthwhile to show that the logics they are studying give rise to square-like structures. Typical examples include the construction of squares for modal logic (Fitting and Mendelsohn 1998; Carnielli and Pizzi 2008), intuitionistic and linear logic (Mélès 2012), structuralist logic (Koslow 1992, 1999), epistemic logic (Lenzen 2012), deontic logic (Moretti 2009b; McNamara 2010) and temporal logic (Rini and Cresswell 2012). Applications of the square to natural language have been explored by linguists such as van der Auwera (1996), Horn (1989, 2012) and Seuren (2010, 2012a,b).

Formally speaking, we will take the Aristotelian square to be a concrete diagram that visualizes an underlying abstract geometry, i.e., a set of logical relations between formulas (relative to some background logical system S). ${ }^{3}$

Definition 1 (Aristotelian geometry) Let $S$ be a logical system, which is assumed to have connectives expressing classical negation $(\neg)$, conjunction $(\wedge)$ and implication

\footnotetext{
1 For the sake of readability, some technical remarks and results have been placed in an appendix; they are not essential for our main line of argumentation.

2 For a more exhaustive historical overview, see Parsons (2006) and Seuren (2010, chapter 5).

3 In this paper, the term 'Aristotelian' is used in a strictly technical sense, to distinguish the Aristotelian geometry and its diagrams from other kinds of geometries and diagrams that will be introduced later. Hence, by calling a certain relation 'Aristotelian' we do not mean to imply that Aristotle himself acknowledged that relation; similarly, by calling a certain diagram 'Aristotelian' we do not mean to imply that Aristotle himsef drew such a diagram, or even defended its validity. (For a detailed account of the historical origins of the square, see Londey and Johanson (1984).) Finally, the discussion of the problems in the Aristotelian geometry in Subsection 3.1 should not be seen as a piece of historical Aristotle scholarship, but rather as the systematic development of a new perspective on some issues in contemporary logic.
} 
$(\rightarrow),{ }^{4}$ and a model-theoretic semantics. Let $\mathscr{L}_{\mathrm{S}}$ be the language of S. The Aristotelian relations for $\mathrm{S}$ are defined as follows: the formulas $\varphi, \psi \in \mathscr{L}_{\mathrm{S}}$ are

$$
\begin{aligned}
& \text { S-contradictory iff } \mathrm{S}=\neg(\varphi \wedge \psi) \quad \text { and } \quad \mathrm{S} \models \neg(\neg \varphi \wedge \neg \psi) \text {, } \\
& \text { S-contrary iff } \mathrm{S}=\neg(\varphi \wedge \psi) \quad \text { and } \quad \mathrm{S} \not \models \neg(\neg \varphi \wedge \neg \psi) \text {, } \\
& \text { S-subcontrary iff } \mathrm{S} \not \models \neg(\varphi \wedge \psi) \quad \text { and } \quad \mathrm{S} \models \neg(\neg \varphi \wedge \neg \psi) \text {, } \\
& \text { in S-subalternation iff } \mathrm{S} \models \varphi \rightarrow \psi \quad \text { and } \mathrm{S} \not \models \psi \rightarrow \varphi \text {. }
\end{aligned}
$$

The Aristotelian geometry for $\mathrm{S}$ is the set $\mathscr{A} \mathscr{G}_{\mathrm{S}}=\{C D, C, S C, S A\}$ of the four Aristotelian relations for $S$ (the abbreviations stand for contradiction, contrariety, subcontrariety and subalternation, respectively).

When the system $S$ is clear from the context, we will often leave it implicit, and simply talk about 'contrary' instead of 'S-contrary', etc. Intuitively, the first three relations - $C D, C$ and $S C$-are defined in terms of whether the formulas can be true together (the $\varphi \wedge \psi$ part) and whether they can be false together (the $\neg \varphi \wedge \neg \psi$ part); ${ }^{5}$ the fourth relation- $S A-$ is defined in terms of truth propagation. ${ }^{6}$

Definition 2 (Aristotelian diagrams) Let $\mathrm{S}$ be a logical system as in Definition 1. An Aristotelian diagram for $\mathrm{S}$ is a diagram that visualizes an edge-labeled graph $G$. The vertices of $G$ are contingent and pairwise non-equivalent formulas $\varphi_{1}, \ldots, \varphi_{n} \in$ $\mathscr{L}_{S} ;{ }^{7}$ the edges of $G$ are labeled by the Aristotelian relations between those formulas, i.e., if $\varphi_{i}$ and $\varphi_{j}$ stand in any Aristotelian relation, then this is visualized according to the code in Figure 1.8

Fig. 1 Code for visually representing the Aristotelian relations

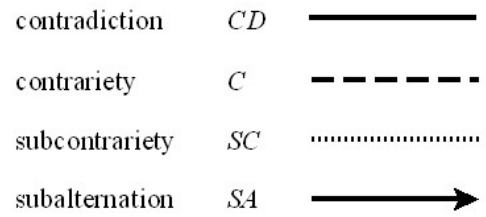

Note that Definition 2 allows only contingent and pairwise non-equivalent formulas to appear in Aristotelian diagrams. The first reason for these restrictions is of a historical nature: classically, squares of oppositions only contained non-equivalent

\footnotetext{
4 It is well-known that in the presence of classical negation, each of $\wedge$ and $\rightarrow$ can be defined in terms of the other: $\varphi \rightarrow \psi=\neg(\varphi \wedge \neg \psi)$, and $\varphi \wedge \psi=\neg(\varphi \rightarrow \neg \psi)$. We will return to this remark in Subsection 3.3.

5 It is well-known that $\neg(\neg \varphi \wedge \neg \psi)$ is equivalent to $\varphi \vee \psi$, but we choose to stick with the first notation, because it more clearly expresses the idea of $\varphi$ and $\psi$ being false together.

6 It should be clear that we do not view the Aristotelian relations in terms of properties of the formulas they relate such as quantity and quality, as is done in many historical studies on Aristotelian logic (Parry and Hacker 1991). For more about this difference, see Demey (2012c, pp. 328-329).

7 So $\mathrm{S} \not \models \varphi_{i}, \mathrm{~S} \not \models \neg \varphi_{i}$, and $\mathrm{S} \not \models \varphi_{i} \leftrightarrow \varphi_{j}$, for $1 \leq i \neq j \leq n$.

8 It follows immediately from Definition 1 that the first three relations are symmetric, and are therefore represented in Figure 1 by lines without arrows. We represent $\varphi$ and $\psi$ being in subalternation by means of an arrow going from $\varphi$ to $\psi$, classically referred to as the 'superaltern' and 'subaltern', respectively.
} 
contingencies. More importantly, although the Aristotelian geometry perfectly allows non-contingencies to enter into multiple Aristotelian relations with other formulas, ${ }^{9}$ those relations will be vacuous and visualizing them would needlessly mess up the diagrams (Sanford 1968). Furthermore, the restriction to pairwise non-equivalent formulas shows that Aristotelian diagrams are essentially semantic entities: like Hasse diagrams, they represent formulas only up to logical equivalence. ${ }^{10}$

The most prototypical Aristotelian diagrams are those which have exactly four vertices, better known as the Aristotelian squares. Figure 2 shows three such Aristotelian squares for fragments of (a) classical propositional logic (CPL), (b) the modal logic S5, and (c) the deontic logic KD. ${ }^{11}$ For example, $p \wedge q$ and $p \vee q$ are in CPLsubalternation $(\mathrm{CPL}=(p \wedge q) \rightarrow(p \vee q)$ and $\mathrm{CPL} \not \forall(p \vee q) \rightarrow(p \wedge q)), \square p$ and $\square \neg p$ are S5-contrary (S5 $\mid=\neg(\square p \wedge \square \neg p)$ and S5 $\mid \models \neg(\neg \square p \wedge \neg \square \neg p)$, and $\mathrm{P} p$ and $\mathrm{P} \neg p$ are KD-subcontrary $(\mathrm{KD} \mid \not \neg(\mathrm{P} p \wedge \mathrm{P} \neg p)$ and $\mathrm{KD} \models \neg(\neg \mathrm{P} p \wedge \neg \mathrm{P} \neg p)$. The modal logic $\mathrm{S} 5$ will be used as a running example throughout this paper. ${ }^{12}$

Fig. 2 Aristotelian squares for (a) CPL, (b) the modal system S5 and (c) the deontic system KD.

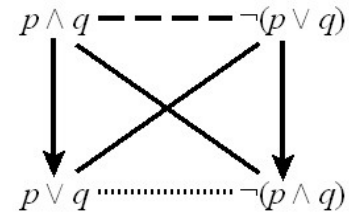

(a)

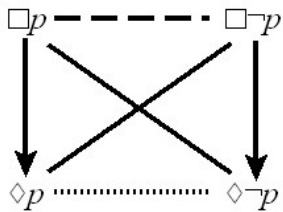

(b)

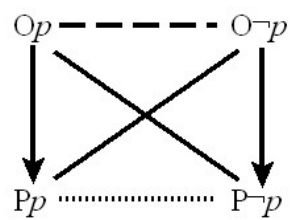

(c)

\subsection{Extensions of the Aristotelian Square}

It should be noted that Definition 2 does not require an Aristotelian diagram to have only 4 formulas as vertices. Unsurprisingly, then, there have been several proposals throughout history to extend the Aristotelian square to more complex diagrams. The most widely known extension is the Aristotelian hexagon proposed by Jacoby (1950, 1960), Sesmat (1951) and Blanché (1952, 1966). A different Aristotelian hexagon was proposed by Czeżowski (1955), although it was already known by the 13thcentury logician William of Sherwood (Kretzmann 1966; Khomskii 2012). While the first type of hexagon is closed under Boolean operators, the second one is not; these, and other, differences are studied in Smessaert (2012a). Further two-dimensional generalizations include the octagons described by Béziau (2003) and Seuren (2010); the

\footnotetext{
9 Tautologies are subaltern and subcontrary to any contingent formula. Conversely, contradictions are superaltern and contrary to any contingent formula.

10 For a more detailed discussion of the connection between Aristotelian diagrams and Hasse diagrams, see Smessaert (2009) and Demey and Smessaert (2014).

11 The operators $\mathrm{O}$ and $\mathrm{P}$ in the deontic square stand for 'obligatory' and 'permitted', respectively.

12 Note that we will not consider squares for the quantifiers, and thus sidestep the notoriously difficult issue of existential import (Chatti and Schang 2013; Parsons 2006; Read 2012a; Seuren 2012a), since the informativity account to be developed here is entirely independent of it.
} 
latter was already known by the 14th-century logician John Buridan (Hughes 1987; Read 2012b). Figure 3 shows two hexagons and an octagon for the modal logic S5. ${ }^{13}$

Fig. 3 (a) Sesmat-Blanché hexagon, (b) Sherwood-Czeżowski hexagon, and (c) Béziau octagon for S5.

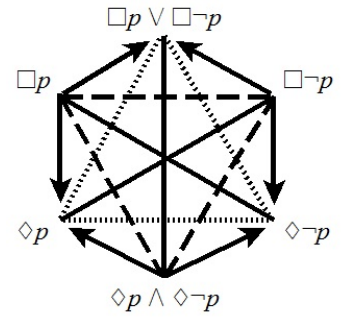

(a)

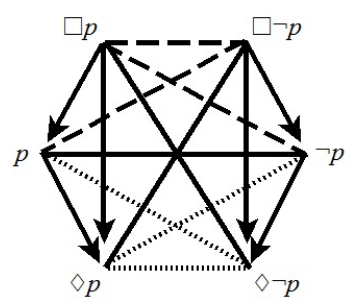

(b)

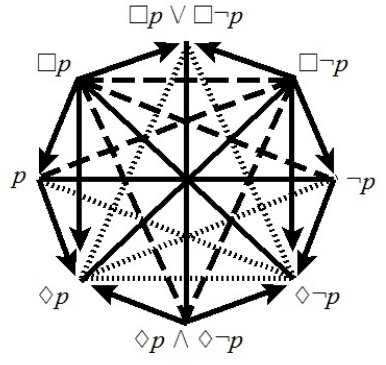

(c)

In recent years, even further generalizations have been proposed, moving from the two-dimensional to the three-dimensional realm. For example, Moretti (2009a) and Chatti and Schang (2013) study two types of Aristotelian cubes, while Smessaert (2009) and Demey (2012c) describe a rhombic dodecahedron, a diagram comprising 14 formulas. Other, related Aristotelian diagrams have been studied by Sauriol (1968) and Moretti (2009a, 2012b).

These larger Aristotelian diagrams seem to be manifestations of a deeper relation between the disciplines of logic and geometry. For example, reflecting upon the logical interpretation of the rhombic dodecahedron, Kauffman (2001, p. 91) writes:

[there exists a] remarkable connection of polyhedral geometry with basic logic [. . . ] One does not expect to find direct connections of the structure of logical speech with the symmetries of Euclidean Geometry. [. . . ] The relationship of logic and geometry demands a deep investigation. This investigation is in its infancy.

In ongoing work, we are developing an exhaustive typology of Aristotelian diagrams, which allows us to classify all the diagrams mentioned above (and many others), and to study their various interrelationships (Smessaert and Demey 2014). The informational account that is developed in this paper, however, is conceptually prior to this typology, so we will not go into it any further.

\footnotetext{
13 Note that Figure 3(a) contains the formula $\diamond p \wedge \diamond \neg p$, which is usually taken to express the (metaphysical) contingency of $p$. This notion of contingency is distinct from the logical notion of contingency that is used in Definition 2 of Aristotelian diagram (see Footnote 7). Although the formula $\square p$ expresses that $p$ is metaphysically necessary, it is itself logically contingent (i.e., $\mathrm{S} 5 \not \forall \square p$ and $\mathrm{S} 5 \not \models \neg \square p$ ), and is thus perfectly allowed to occur in Aristotelian diagrams. Finally, note that the octagon in Figure 3(c) can be seen as the 'sum' of the hexagons in (a) and (b).
} 


\subsection{The Success of the Aristotelian Square}

From a theoretical perspective, there does not seem to be any fundamental difference between the Aristotelian square and its extensions: both the square and its extensions are just examples of Aristotelian diagrams (cf. Definition 2). In practice, however, there is a major difference in popularity: while the square is nearly universally known among logicians and formal linguists, many of the larger diagrams are only known by a few specialists studying them. ${ }^{14}$ This might partially be explained by the relative recency of their discovery; however, even the hexagons that were already being investigated in the 1950s have never been able to attract much attention (despite having various interesting properties, as shown by Smessaert 2012a).

Another explanation of the square's success is based on the intuition that this diagram is highly informative. ${ }^{15}$ Unfortunately, this intuition is quite vague; e.g. what does 'informative' mean here?-and are the larger diagrams then supposed to be less informative than the square? In the remainder of this paper, however, we will argue that this intuition is essentially on the right track: we will develop a formal, well-motivated account of information in (Aristotelian and other) geometries and diagrams, and then use it to show that the square is indeed more informative than many of the more complex diagrams.

\section{The Logical Geometries of Opposition and Implication}

This section introduces two new logical geometries in addition to the classical Aristotelian geometry. Subsection 3.1 discusses some problems that have traditionally been associated with the Aristotelian geometry. Subsection 3.2 defines the opposition geometry and the implication geometry, as well as their associated diagrams. Subsection 3.3 shows that these geometries not only solve the problems of the Aristotelian geometry, but also have several independent motivations.

\subsection{Problems with the Aristotelian Geometry}

The Aristotelian geometry, as introduced in Definition 1, seems to suffer from a number of problems. For starters, this geometry does not induce a partition on the formula-pairs, and thus fails to provide a full organization of logical space. On the one hand, the Aristotelian relations are not mutually exclusive: as was already discussed in Subsection 2.1, there exist pairs of formulas that simultaneously stand in

\footnotetext{
14 This does not mean that these extensions do not have any applications at all. For example, Horn (1990) uses various hexagons to study Gricean maxims and conversational implicatures, while Jaspers (2012) uses the Sesmat-Blanché hexagon to analyze the structure of the color categories from a logical, linguistic and cognitive perspective.

15 For example, this intuition seems to be implicit in the following remarks: "familiarity with the square is useful for logicians today as a kind of lingua franca, when adapted as a shorthand to express logical relations in specialized applied logics with specialized domains" (Jacquette 2012, p. 81), and "the square $[\ldots]$ is a compact way of representing various logical relations between formulas, and thus serves as an illustration of the underlying logic's expressive and deductive powers" (Demey 2012c, p. 314).
} 
two Aristotelian relations. For example, the formulas $p \wedge \neg p$ and $p$ are both contrary and in subalternation, whereas $p$ and $p \vee \neg p$ are both subcontrary and in subalternation. ${ }^{16}$ On the other hand, the Aristotelian geometry is not exhaustive either: some pairs of formulas - for example, $p$ and $\diamond p \wedge \diamond \neg p$ —stand in no Aristotelian relation whatsoever. A particular subclass of such pairs results from the Aristotelian relations' irreflexivity on contingent formulas: no contingent formula stands in any Aristotelian relation whatsoever to itself.

Most importantly, however, the Aristotelian geometry is inherently based on a certain conceptual confusion, which is visible in the relations' definitions and which will turn out to have far-reaching consequences. Whereas the first three Aristotelian relations (contradiction, contrariety and subcontrariety) are characterized in terms of the related formulas possibly being true/false together, the fourth relation (subalternation) is characterized in terms of truth propagation. These two notions are conceptually independent: the former is commutative $(\varphi$ and $\psi$ can be true together iff $\psi$ and $\varphi$ can be true together), whereas the latter is directional (truth is propagated from $\varphi$ to $\psi$ ). The commutativity of 'together' is captured by the conjunctions in the definitions of the first three relations, which are therefore symmetrical: for $R=C D$, $C$ and $S C$, we have $R(\varphi, \psi)$ iff $R(\psi, \varphi)$. By contrast, the directionality of 'propagation' is captured by the implications in the definition of the fourth relation, which is therefore asymmetrical: if $S A(\varphi, \psi)$, then not $S A(\psi, \varphi)$.

\subsection{Defining the Opposition and Implication Geometries}

We have just argued that the first three Aristotelian relations are conceptually independent from the fourth one. These three relations are all based on the idea of the related formulas possibly being true/false together. Combinatorially speaking, this idea leads to four separate cases:

1. the related formulas cannot be true together, and cannot be false together,

2. the related formulas cannot be true together, but can be false together,

3. the related formulas can be true together, but cannot be false together,

4. the related formulas can be true together, and can be false together.

The first three cases correspond exactly with the Aristotelian relations of contradiction, contrariety and subcontrariety, respectively. The fourth case, however, does not correspond with any Aristotelian relation. The relation corresponding to this case will be called non-contradiction (Smessaert 2009, p. 310). ${ }^{17}$

\footnotetext{
16 Note that both examples involve a non-contingent formula. This is not a coincidence: if we focus on contingent formulas, the Aristotelian relations are mutually exclusive (Demey 2012c, Lemma 3.2).

17 Non-contradiction is clearly different from the Aristotelian relation of subalternation. First of all, there exist pairs of formulas-such as $(p, \neg \neg p)$ and $(p, q)$-which are in non-contradiction, but not in subalternation. Furthermore, if two contingent formulas $\varphi$ and $\psi$ are in subalternation, they will also be in non-contradiction, but that characterization would miss the key point that the truth values of $\varphi$ and $\psi$ are not independent (if $\varphi$ is true, then $\psi$ has to be true as well).
} 
In the light of these observations, it is natural to remove the subalternation relation from the Aristotelian geometry, and to replace it with the non-contradiction relation. The new geometry that is thus obtained, will be called the 'opposition geometry'. ${ }^{18}$

Definition 3 (opposition geometry) Let $S$ be a logical system as in Definition 1 . The opposition relations for $\mathrm{S}$ are defined as follows: the formulas $\varphi, \psi \in \mathscr{L}_{\mathrm{S}}$ are

\begin{tabular}{|c|c|c|c|c|}
\hline -contradictory & iff & $\mathrm{S} \models \neg(\varphi \wedge \psi)$ & and & $\mathrm{S}=\neg(\neg \varphi$ \\
\hline & & $\mathrm{S} \models \neg(\varphi \wedge \psi)$ & and & $\psi$ \\
\hline rary & & $\mathrm{S} \not \models \neg(\varphi \wedge \psi)$ & and & $\neg \neg \psi$ \\
\hline -non-contradictory & if & $\mathrm{S} \not \models \neg(\varphi \wedge \psi)$ & and & \\
\hline
\end{tabular}

The opposition geometry for $\mathrm{S}$ is the set $\mathscr{O} \mathscr{G}_{\mathrm{S}}=\{C D, C, S C, N C D\}$ of the four opposition relations for $\mathrm{S}$ (the abbreviation $N C D$ stands for non-contradiction).

Consider again the relation of subalternation, which we have just removed from the Aristotelian geometry to obtain the opposition geometry. This relation is based on the idea of truth propagation (entailment), with truth being propagated from the left formula $(\varphi)$ to the right one $(\psi)$, i.e., $\varphi$ entails $\psi$, and not vice versa. Combinatorially speaking, there are four 'directions' of truth propagation:

1. $\varphi$ entails $\psi$, and $\varphi$ is entailed by $\psi$,

2. $\varphi$ entails $\psi$, but $\varphi$ is not entailed by $\psi$,

3. $\varphi$ does not entail $\psi$, but $\varphi$ is entailed by $\psi$,

4. $\varphi$ does not entail $\psi$, and $\varphi$ is not entailed by $\psi$.

The second case corresponds to the relation of subalternation, which will also be called left-implication (because truth is propagated from left to right). Continuing this naming convention, the relations corresponding to cases 1,3 and 4 will be called bi-implication, right-implication and non-implication, respectively. Together, these four relations constitute the implication geometry:

Definition 4 (implication geometry) Let $\mathrm{S}$ be a logical system as in Definition 1. The implication relations for $\mathrm{S}$ are defined as follows: the formulas $\varphi, \psi \in \mathscr{L}_{\mathrm{S}}$ are in
S-bi-implication
iff $\mathrm{S}=\varphi \rightarrow \psi \quad$ and $\mathrm{S} \models \psi \rightarrow \varphi$,
S-left-implication
iff $\mathrm{S}=\varphi \rightarrow \psi \quad$ and $\mathrm{S} \not \models \psi \rightarrow \varphi$,
S-right-implication
iff $\mathrm{S} \not \models \varphi \rightarrow \psi \quad$ and $\mathrm{S} \models \psi \rightarrow \varphi$,
S-non-implication
iff $\mathrm{S} \not \models \varphi \rightarrow \psi \quad$ and
$\mathrm{S} \not=\psi \rightarrow \varphi$.

The implication geometry for $S$ is the set $\mathscr{I} \mathscr{G}_{\mathrm{S}}=\{B I, L I, R I, N I\}$ of the four implication relations for $\mathrm{S}$ (the abbreviations stand for bi-, left-, right- and non-implication, respectively).

\footnotetext{
18 'Opposition geometry' (Definition 3) is a technical term, on a par with 'Aristotelian geometry' (Definition 1) and 'implication geometry' (Definition 4), and should thus not be confused with the general framework of oppositional geometry developed by Moretti (2012b). Furthermore, note that Definition 3 is similar in spirit to Moretti (2009a, 2012a) and Schang (2012c)'s 'question-answer semantics'; however, they propose this as a semantics for the Aristotelian geometry, and thus fail to fully distinguish between the relations of subalternation and non-contradiction (recall Footnote 17). Recently, however, Schang (2012a, 2013) has shown that subalternation can be understood in terms of 'contradictories of contraries' (in the sense that $S A(\varphi, \psi)$ iff there is a formula $\theta$ such that $C(\varphi, \theta)$ and $C D(\theta, \psi)$; see item $6 \mathrm{~b}$ of our Lemma 3), and used this fact to argue that subalternation is an Aristotelian relation after all.
} 
Remark 1 The opposition and implication relations are all defined by means of the propositional functions $\Delta_{1}-\Delta_{4}$ :

- $\Delta_{1}(\varphi, \psi):=(\varphi \wedge \psi)$,

- $\Delta_{2}(\varphi, \psi):=(\varphi \wedge \neg \psi)$,

- $\Delta_{3}(\varphi, \psi):=(\neg \varphi \wedge \psi)$,

- $\Delta_{4}(\varphi, \psi):=(\neg \varphi \wedge \neg \psi)$.

The opposition relations are defined in terms of (the negations of) $\Delta_{1}$ and $\Delta_{4}$. Similarly, recalling that $\alpha \rightarrow \beta$ is equivalent to $\neg(\alpha \wedge \neg \beta)$, it should be clear that the implication relations are defined in terms of (the negations of) $\Delta_{2}$ and $\Delta_{3}$. Each of these functions provides a complete description of the world with respect to $\varphi$ and $\psi$, and is thus related to Carnap (1947)'s notion of state description. ${ }^{19}$ These propositional functions jointly partition logical space, as is illustrated in Figure 4. The opposition and implication relations holding between $\varphi$ and $\psi$ are determined by which of the regions $\left[\left[\Delta_{i}(\varphi, \psi)\right]\right]$ are empty; this corresponds to the fact that the opposition and implication relations are defined in terms of the negations of $\Delta_{i}$.

Fig. 4 The partition of logical space $\left(\mathscr{C}_{S}\right)$ induced by the propositions $\Delta_{i}(\varphi, \psi)$

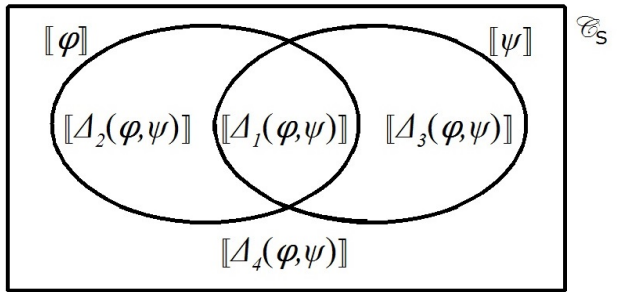

Remark 2 The opposition and implication geometries jointly solve the problems of the Aristotelian geometry. First of all, it is easy to show that both the opposition relations and the implication relations are mutually exclusive and jointly exhaustive: each pair of formulas stands in one and only one opposition relation and in one and only one implication relation. More importantly, the conceptual confusion underlying the Aristotelian geometry is dissolved: the opposition geometry is uniformly based on the notion of 'possibly being true/false together' (its relations are defined in terms of $\Delta_{1}$ and $\Delta_{4}$ ), and the implication geometry is uniformly based on the notion of 'truth propagation' (its relations are defined in terms of $\Delta_{2}$ and $\Delta_{3}$ ).

The opposition and implication geometries are visualized by opposition and implication diagrams, in exactly the same way as the Aristotelian geometry is visualized by Aristotelian diagrams (recall Definition 2).

Definition 5 (opposition and implication diagrams) Let $\mathrm{S}$ be a logical system as in Definition 1. An opposition diagram (resp. implication diagram) for $\mathrm{S}$ is a diagram

19 Obviously, the indices come from the canonical way of displaying a truth table for a binary connective. 
that visualizes an edge-labeled graph $G$. The vertices of $G$ are contingent and pairwise non-equivalent formulas $\varphi_{1}, \ldots, \varphi_{n} \in \mathscr{L}_{\mathrm{S}}$; the edges of $G$ are labeled by the opposition relations (resp. implication relations) between those formulas, i.e., if $\varphi_{i}$ and $\varphi_{j}$ stand in any opposition (resp. implication) relation, then this is visualized according to the code in Figure 5.

Fig. 5 Code for visually representing the opposition and implication relations

$\begin{array}{lllll}\text { contradiction } & C D & \text { bi-implication } & B I \\ \text { contrariety } & C & \text { left-implication } & L I \\ \text { subcontrariety } & S C & \text { right-implication } & R I \\ \text { non-contran............ } & \text { non-implication } & N I\end{array}$

Since the two new geometries were obtained by disentangling the Aristotelian geometry, several relations occur in both the Aristotelian geometry and one of the new geometries; obviously, opposition/implication diagrams visualize these relations in the same way as Aristotelian diagrams (compare the codes in Figures 1 and 5). Furthermore, some opposition and implication relations are visualized in the same way (in particular, solid black lines for $C D$ as well as $B I$, or solid grey lines for $N C D$ as well as $N I) .{ }^{20}$ However, this should not cause any confusion, because a diagram for a given geometry visualizes only relations belonging to that geometry (for example, a solid grey line in an opposition diagram can only represent $N C D$ ). Finally, the six symmetric opposition and implication relations are represented by lines without arrows; the asymmetric relations of $L I$ and $R I$ are represented by arrows, with the arrow going from the relation's first argument to its second argument. Thus, $\operatorname{LI}(\varphi, \psi)$ and $R I(\varphi, \psi)$ are visualized as $\varphi \rightarrow \psi$ and $\varphi \longrightarrow \psi$, respectively. ${ }^{21}$

These visual properties are illustrated in Figure 6, which shows an Aristotelian diagram, an opposition diagram and an implication diagram for one and the same fragment of S5-formulas: $\{\square p, \square \neg p, \diamond p\}$.

\subsection{Motivating the New Geometries}

In the previous subsection we introduced the opposition and implication geometries, and showed that they jointly solve the problems of the Aristotelian geometry. In this subsection, we will show that they also have a number of (historical and technical) independent motivations.

\footnotetext{
20 The contrast within both geometries between (three kinds of) black lines on the one hand and a grey line on the other is motivated by informativity considerations that will be presented later in the paper.

21 The arrow's head indicates the direction of truth propagation. In the case of $L I$, this direction matches the direction of the arrow itself, but in the case of $R I$, they differ. For example, $L I(\square p, p)$ is visualized as $\square p \rightarrow p$, because both the $L I$-relation and truth propagation go from $\square p$ to $p$; however, $R I(\diamond p, p)$ is visualized as $\diamond p \longrightarrow p$, because the RI-relation goes from $\nabla p$ to $p$, but truth is propagated from $p$ to $\diamond p$.
} 
Fig. 6 (a) Aristotelian diagram, (b) opposition diagram, and (c) implication diagram for an S5-fragment

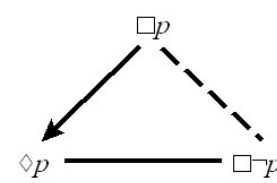

(a)

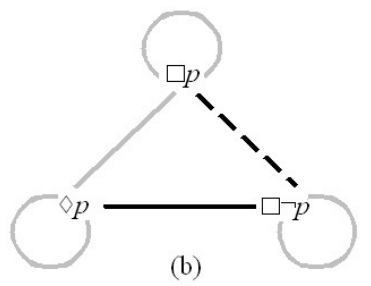

(b)

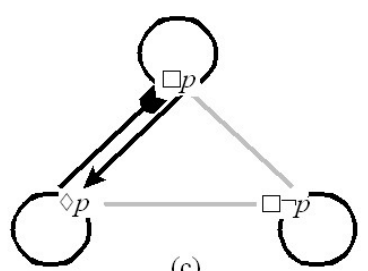

(c)

Historical Precursors. The two new geometries are firmly rooted in a long-standing tradition of discussions about the exact nature of the Aristotelian relations. For example, already in the second century AD, Apuleius observed that the relations of contradiction, contrariety and subcontrariety are all based on the notion of 'possibly being true/false together', which he called 'pugna'. Subalternation, however, falls outside the scope of this notion: "[u]nder the truth-functional perspective of pugnae we learn quickly that a-i and e-o [i.e., subaltern formulas] are neither in pugna perfecta [CD], nor in pugna dividua [C/SC], but they are in no pugna whatsoever" (Gombocz 1990, p. 126). If Apuleius' notion of 'no pugna whatsoever' is viewed as non-contradiction, his pugna-perspective clearly anticipates the opposition geometry.

Furthermore, Correia (2012, p. 42) convincingly argues that there are two complementary perspectives on the square: as a theory of negation and as a theory of logical consequence. Both perspectives have been discussed in separate textual traditions of Aristotle's work: the former is mainly found in commentaries on De Interpretatione, while the latter is central in commentaries on Prior Analytics. As Correia points out, these perspectives are based on "two kinds of logical relations that commentators distinguished in their comments on the square: relations of opposition [CD, C, SC] and relations of the parts and the whole [SA/LI]" (2012, p. 47). Hence, the negation- and consequence-perspectives (with their underlying logical relations) clearly anticipate the opposition and implication geometries, respectively.

Internal and External Structure. The two new geometries are highly structured, both internally and externally (i.e., with respect to each other). We will first look at the geometries' internal structure. Since the opposition geometry is based on the commutative notion of 'together', its relations are all symmetric. The implication geometry, however, is based on the directional notion of 'truth propagation'; if the direction of truth propagation is reversed, the roles of left-to-right implication $(L I)$ and right-toleft implication $(R I)$ are changed around (the 'neutral' relations of both-way implication $(B I)$ and neither-way implication (NI) are left untouched). This is summarized in the following lemma.

Lemma 1 For all formulas $\varphi, \psi \in \mathscr{L}_{\mathrm{S}}$, the following hold:

\begin{tabular}{|c|c|c|c|c|c|c|c|}
\hline a) & $C D(\varphi, \psi)$ & iff & $C D(\psi, \varphi)$ & $1 b)$ & $B I(\varphi, \psi)$ & iff & $B I(\psi$ \\
\hline a) & $C(\varphi, \psi)$ & iff & $C(\psi, \varphi)$ & 2b) & $L I(\varphi, \psi)$ & iff & $R I(\psi, \varphi)$ \\
\hline & $S C(\varphi, \psi)$ & iff & $S C(\psi, \varphi)$, & $3 b)$ & $R I(\varphi, \psi)$ & iff & $L I(\psi, \varphi)$, \\
\hline$a$ & $N C D(\varphi, \psi)$ & iff & $N C D(\psi, \varphi)$ & 4b) & $N I(\varphi, \psi)$ & iff & $N I(\psi, \varphi$ \\
\hline
\end{tabular}


Proof All items follow trivially from Definitions 3 and 4.

If we use $\mathscr{G}_{\mathrm{S}}$ to denote the set of all opposition and implication relations ( $\mathscr{G}_{\mathrm{S}}:=$ $\left.\mathscr{O} \mathscr{G}_{\mathrm{S}} \cup \mathscr{I} \mathscr{G}_{\mathrm{S}}\right){ }^{22}$ this lemma can be rephrased in a slightly more compact way. The advantages of this rephrasing will become clear later on.

Corollary 1 There exists a mapping $F: \mathscr{G} \rightarrow \mathscr{G}$ such that for all relations $R \in \mathscr{G}$, it holds for all $\varphi, \psi \in \mathscr{L}_{\mathrm{S}}$ that $R(\varphi, \psi)$ iff $F(R)(\psi, \varphi)$.

Proof The definition of $F$ can be straightforwardly 'read off' from Lemma 1, i.e., put $F(C D):=C D, F(C):=C, F(S C):=S C, F(N C D):=N C D, F(B I):=B I, F(L I):=$ $R I, F(R I):=L I$, and $F(N I):=N I$.

Another, independent way in which the new geometries are internally structured, is that if two formulas stand in some opposition (resp. implication) relation, their negations stand in some opposition (resp. implication) relation as well. Details can be found in the following lemma and corollary.

Lemma 2 For all formulas $\varphi, \psi \in \mathscr{L}_{\mathrm{S}}$, the following hold:

\begin{tabular}{|c|c|c|c|c|c|c|}
\hline$C D(\varphi, \psi)$ & iff & $C D(\neg \varphi, \neg \psi)$, & 1b) & $B I(\varphi, \psi)$ & iff & $B I(\neg \varphi, \neg \psi)$ \\
\hline$C(\varphi, \psi)$ & iff & $S C(\neg \varphi, \neg \psi)$, & $2 b)$ & $L I(\varphi, \psi)$ & iff & $R I$ \\
\hline$S C(\varphi, \psi)$ & iff & $C(\neg \varphi, \neg \psi)$, & $3 b)$ & $R I(\varphi, \psi)$ & iff & $L I(\neg \varphi, \neg \psi$ \\
\hline$N C D(\varphi, \psi)$ & iff & $N C D(\neg \varphi, \neg \psi)$, & $4 b)$ & $N I(\varphi, \psi)$ & iff & $N I(\neg \varphi,-$ \\
\hline
\end{tabular}

Proof All items follow trivially from Definitions 3 and 4.

Corollary 2 There exists a mapping $N 12: \mathscr{G} \rightarrow \mathscr{G}$ such that for all relations $R \in \mathscr{G}$, it holds for all $\varphi, \psi \in \mathscr{L}_{\mathrm{S}}$ that $R(\varphi, \psi)$ iff $N 12(R)(\neg \varphi, \neg \psi)$.

Proof As before, the definition of N12 can be 'read off' from Lemma 2.

It should be noted that for all $R \in \mathscr{G}$, it holds that $F(N 12(R))=N 12(F(R))$; we can thus define the mapping $F N 12: \mathscr{G} \rightarrow \mathscr{G}$ by putting $F N 12:=F \circ N 12=N 12 \circ F$. If we use $I d$ to note the identity mapping on $\mathscr{G}$, the internal structure of the opposition and implication geometries can be summarized as follows:

Remark 3 The set $\{I d, F, N 12, F N 12\}$ is closed under composition (o), and forms a group that acts faithfully on $\mathscr{G}$. This group is isomorphic to the Klein four-group. The separate geometries $\mathscr{O} \mathscr{G}$ and $\mathscr{I} \mathscr{G}$ are invariant under this group action. More details can be found in Remark 7 in the appendix. ${ }^{23}$

We have argued above that the opposition and implication geometries are conceptually independent: the former is based on the notion of 'possibly being true/false together', while the latter is based on the notion of 'truth propagation'. This does not imply, however, that there are absolutely no connections between both geometries. Consider, for example, the opposition relation of contrariety. If $C(\varphi, \psi)$, then $\varphi$ and $\psi$ cannot be true together, which is, by itself, a 'directionless' situation. However, we can impose a direction upon it, in two complementary ways:

\footnotetext{
22 Note that this set includes the original Aristotelian relations, i.e., $\mathscr{A} \mathscr{G} \subseteq \mathscr{G}$.

23 For more background on group theory, see Rotman (1995), in particular p. 55ff. and p. 345ff.
} 
- If the first formula $(\varphi)$ is true, then the second one $(\psi)$ has to be false (because otherwise both formulas would be true together after all).

- If the second formula $(\psi)$ is true, then the first one $(\varphi)$ has to be false (because otherwise both formulas would be true together after all). ${ }^{24}$

It is easy to see that these two ways of symmetry breaking correspond exactly to $L I(\varphi, \neg \psi)$ and $R I(\neg \varphi, \psi)$, and have thus taken us to the implication geometry. The following lemma lists similar ways in which oppositional facts can be expressed using implication relations, and vice versa. ${ }^{25}$

Lemma 3 For all formulas $\varphi, \psi \in \mathscr{L}_{\mathrm{S}}$, the following hold:

$\begin{array}{lrlrlrlr}1 a) & C D(\varphi, \psi) & \text { iff } & B I(\neg \varphi, \psi), & 1 b) & C D(\varphi, \psi) & \text { iff } & B I(\varphi, \neg \psi), \\ 2 a) & C(\varphi, \psi) & \text { iff } & R I(\neg \varphi, \psi), & 2 b) & C(\varphi, \psi) & \text { iff } & L I(\varphi, \neg \psi), \\ 3 a) & S C(\varphi, \psi) & \text { iff } & L I(\neg \varphi, \psi), & 3 b) & S C(\varphi, \psi) & \text { iff } & R I(\varphi, \neg \psi), \\ 4 a) & N C D(\varphi, \psi) & \text { iff } & N I(\neg \varphi, \psi), & 4 b) & N C D(\varphi, \psi) & \text { iff } & N I(\varphi, \neg \psi), \\ 5 a) & B I(\varphi, \psi) & \text { iff } & C D(\neg \varphi, \psi), & 5 b) & B I(\varphi, \psi) & \text { iff } & C D(\varphi, \neg \psi), \\ 6 a) & L I(\varphi, \psi) & \text { iff } & S C(\neg \varphi, \psi), & 6 b) & L I(\varphi, \psi) & \text { iff } & C(\varphi, \neg \psi), \\ 7 a) & R I(\varphi, \psi) & \text { iff } & C(\neg \varphi, \psi), & 7 b) & R I(\varphi, \psi) & \text { iff } & S C(\varphi, \neg \psi), \\ 8 a) & N I(\varphi, \psi) & \text { iff } & N C D(\neg \varphi, \psi), & 8 b) & N I(\varphi, \psi) & \text { iff } & N C D(\varphi, \neg \psi) .\end{array}$

Proof All items follow trivially from Definitions 3 and 4.

Corollary 3 There exist mappings $N 1, N 2: \mathscr{G} \rightarrow \mathscr{G}$ such that for all relations $R \in \mathscr{G}$, the following holds for all $\varphi, \psi \in \mathscr{L}_{\mathrm{S}}$ :

a) $R(\varphi, \psi)$ iff $N 1(R)(\neg \varphi, \psi)$

b) $R(\varphi, \psi)$ iff $N 2(R)(\varphi, \neg \psi)$.

Proof The definitions of $N 1$ and $N 2$ can be 'read off' from the $a$ - and $b$-series of items, respectively, in Lemma 3.

These mappings $N 1$ and $N 2$ are obviously related to the mapping $N 12$ defined above: $N 12=N 1 \circ N 2=N 2 \circ N 1$. If we define mappings $F N 1, F N 2: \mathscr{G} \rightarrow \mathscr{G}$ by $F N 1=F \circ N 1$ and $F N 2=F \circ N 2$, the close relationship between the opposition and implication geometries can be summarized as follows:

Remark 4 The set $\{I d, N 1, N 2, N 12, F, F N 1, F N 2, F N 12\}$ is closed under composition, and forms a group that acts faithfully on $\mathscr{G}$. This group is isomorphic to the dihedral group of order 8 . More details can be found in Remark 8 in the appendix. ${ }^{26}$

\footnotetext{
24 These facts were already known by the 13th-century logician Peter of Spain, who called them the 'law of contraries' (Horn 2010).

25 Lemma 3 consists of an $a$ - and a $b$-series, which describe the effects of negating the first, resp. the second argument of a given relation. The symmetry breaking/creating required to connect the opposition and implication geometries is manifested in the fact that exactly one argument is negated. This is to be contrasted with Lemma 2, in which both arguments are negated, and the geometries are kept apart (opposition relations are connected with opposition relations, implication relations with implication relations).

26 It should be emphasized that the rich structure of the opposition and implication geometries does not primarily consist in the individual items of Lemmas 1-3, but rather in the fact that they interact with each other in interesting ways. These interactions can concisely be described using the language of group theory, as illustrated in Remarks 3-4 and Remarks 7-8.
} 
Geometries and Connectives. There are 4 opposition relations and 4 implication relations, and thus $4 \times 4=16$ possible combinations of an opposition and an implication relation. On the other hand, it is well-known that there are $2^{4}=16$ binary, truth-functional connectives (Enderton 2001, pp. 50-51). We will now show that this numerical equality is not a coincidence, because there exists a canonical correspondence between pairs of opposition and implication relations and binary connectives.

Each binary, truth-functional connective $\bullet$ can be identified with its truth table, i.e., with the 4-tuple $\left(\bullet_{1}, \bullet_{2}, \bullet_{3}, \bullet_{4}\right) \in\{0,1\}^{4}$, where $\bullet_{i}$ is the truth value of the formula $\varphi \bullet \psi$ on row $i$, i.e., in case $\Delta_{i}(\varphi, \psi)$ is true. Formally, this means that

$$
\begin{aligned}
& \text { if } \bullet=1 \text {, then } \mathrm{S} \models \Delta_{i}(\varphi, \psi) \rightarrow(\varphi \bullet \psi) \text {, } \\
& \text { if } \bullet=0 \text {, then } \mathrm{S} \models \Delta_{i}(\varphi, \psi) \rightarrow \neg(\varphi \bullet \psi) \text {. }
\end{aligned}
$$

For example, conjunction is $\wedge=(1,0,0,0)$, while (inclusive) disjunction is $\vee=$ $(1,1,1,0)$. This identification between connectives and their truth tables is used in the following definition: ${ }^{27}$

Definition 6 Given an opposition relation $R \in \mathscr{O} \mathscr{G}$ and an implication relation $S \in$ $\mathscr{I} \mathscr{G}$, we define the binary, truth-functional connective $\bullet^{(R, S)}$ by putting, for $1 \leq i \leq 4$ :

$\bullet_{i}^{(R, S)}:= \begin{cases}0 & \text { if for all } \varphi, \psi \in \mathscr{L}_{S} \text { such that } R(\varphi, \psi) \text { and } S(\varphi, \psi): \models \neg \Delta_{i}(\varphi, \psi), \\ 1 & \text { if there exist } \varphi, \psi \in \mathscr{L}_{S} \text { such that } R(\varphi, \psi) \text { and } S(\varphi, \psi): \forall \neg \Delta_{i}(\varphi, \psi) .\end{cases}$

As noted in Remark 1, the opposition relation $R$ is defined in terms of $\neg \Delta_{1}$ and $\neg \Delta_{4}$, and thus determines the values of $\bullet_{1}^{(R, S)}$ and $\bullet_{4}^{(R, S)}$; similarly, the implication relation $S$ is defined in terms of $\neg \Delta_{2}$ and $\neg \Delta_{3}$, and thus determines the values of $\bullet_{2}^{(R, S)}$ and $\bullet{ }_{3}^{(R, S)}$. In total, the pair $(R, S) \in \mathscr{O} \mathscr{G} \times \mathscr{I} \mathscr{G}$ yields the connective $\bullet(R, S)$. For example, $(S C, N I)$ yields the connective $\bullet(S C, N I)=(1,1,1,0)=\vee$, and $(S C, L I)$ yields the connective $\bullet(S C, L I)=(1,0,1,0)$.

Definition 6 thus associates each pair of an opposition relation $R$ and an implication relation $S$ with a truth-functional, binary connective $\bullet^{(R, S)}$. It is easy to see that this mapping $(R, S) \longmapsto \bullet^{(R, S)}$ is a bijection:

- it is injective: for all opposition relations $R, R^{\prime}$ and implication relations $S, S^{\prime}$, $\bullet^{(R, S)}=\bullet^{\left(R^{\prime}, S^{\prime}\right)}$ implies that $R=R^{\prime}$ and $S=S^{\prime}$,

- it is surjective: for every binary, truth-functional connective $\bullet$, there exist an opposition relation $R$ and an implication relation $S$ such that $\bullet=\bullet \bullet^{(R, S)}$.

The mere existence of a bijection between $\mathscr{O} \mathscr{G} \times \mathscr{I} \mathscr{G}$ and the set of all truth-functional, binary connectives should come as no surprise, since we already knew that both sets have the same cardinality (viz., 16). Theorem 1 below states that the bijection described in Definition 6 is canonical, and thus provides a positive answer to the

\footnotetext{
27 Definition 6 might look cumbersome, because it involves quantifying over formulas. However, it follows immediately from Definitions 3-4 that if $R(\varphi, \psi), S(\varphi, \psi), R\left(\varphi^{\prime}, \psi^{\prime}\right)$ and $S\left(\varphi^{\prime}, \psi^{\prime}\right)$, then for $1 \leq$ $i \leq 4: \models \neg \Delta_{i}(\varphi, \psi) \Leftrightarrow \models \neg \Delta_{i}\left(\varphi^{\prime}, \psi^{\prime}\right)$. This shows that the quantification over formulas in Definition 6 is 'innocent': if there exists at least one pair of formulas $(\varphi, \psi)$ standing in the relations $R$ and $S$ for which it holds that $\models \neg \Delta_{i}(\varphi, \psi)$, then this holds for all such pairs of formulas.
} 
question whether "each [binary] logical connective corresponds to a relation of opposition" (Schang 2012b, p. 152) - at least, if Schang's 'relation of opposition' is re-interpreted as 'pair of an opposition relation and an implication relation'.

Theorem 1 Consider an opposition relation $R \in \mathscr{O} \mathscr{G}$ and an implication relation $S \in \mathscr{I} \mathscr{G}$. Then for all formulas $\varphi, \psi \in \mathscr{L}_{\mathrm{S}}$, the following holds:

$$
\text { if } R(\varphi, \psi) \text { and } S(\varphi, \psi) \text {, then } \mathrm{S} \models \varphi \bullet^{(R, S)} \psi \text {. }
$$

Proof Let $\varphi, \psi \in \mathscr{L}_{S}$ be arbitrary formulas and suppose that $R(\varphi, \psi)$ and $S(\varphi, \psi)$. Let $\mathbb{M}$ be an arbitrary model (of the semantics of the system $S$ ); we will show that $\mathbb{M} \models \varphi \bullet(R, S) \psi$. By definition of the propositional functions $\Delta_{i}$, there exists exactly one $i \in\{1,2,3,4\}$ such that $\mathbb{M} \models \Delta_{i}(\varphi, \psi)$. Hence $\forall \neg \Delta_{i}(\varphi, \psi)$, and thus it follows by Definition 6 that $\bullet_{i}^{(R, S)}=1$. Given the connection between a connective and its truth table—as formally expressed by (1) and (2)—, it thus follows that $=\Delta_{i}(\varphi, \psi) \rightarrow$ $\left(\varphi \bullet^{(R, S)} \psi\right)$. Hence, $\mathbb{M}=\Delta_{i}(\varphi, \psi)$ entails that $\mathbb{M} \models \varphi \bullet^{(R, S)} \psi$.

Consider, for example, the relations $S C$ and $N I$, and recall that $\bullet(S C, N I)=(1,1,1,0)$ $=\vee$. Theorem 1 now states that for any formulas $\varphi, \psi$ standing in these relations, it holds that $=\varphi \vee \psi$. For another example, consider the relations $S C$ and $L I$, and recall that $\bullet^{(S C, L I)}=(1,0,1,0)$; Theorem 1 now states that for any formulas $\varphi, \psi$ standing in these relations, it holds that $=\psi .^{28}$

The correspondence established above is certainly not the only connection between the binary, truth-functional connectives and logical geometry. For example, several authors have noted that these connectives can be used to decorate a rhombic dodecahedron (Zellweger 1997; Kauffman 2001) and related diagrams (Sauriol 1968; Luzeaux et al 2008; Moretti 2009a; Dubois and Prade 2012). Such diagrams visualize the Aristotelian relations that hold between propositions of the form $p \bullet q$ and $p \circ q$, where $\bullet$ and $\circ$ are binary, truth-functional connectives. Hence, the connectives appear at the object level: they are (inside) the relata, i.e., the concrete formulas standing in the Aristotelian relations. Theorem 1, however, is of a fundamentally different nature, because it operates on the metalevel: it does not link the connectives with the relata of the opposition and implication relations, but rather with these relations themselves.

Additionally, Theorem 1 immediately leads to Theorem 2 below, which states that contingent formulas can stand in only 7 out of the 16 combinatorially possible pairs of opposition and implication relations. This restriction will turn out to have a number of applications in the remainder of the paper.

Theorem 2 Consider arbitrary formulas $\varphi, \psi \in \mathscr{L}_{\mathrm{S}}$, and suppose that $\varphi$ and $\psi$ are contingent. Then $\varphi$ and $\psi$ stand in one of the following 7 pairs of relations:

$$
\begin{array}{lr}
(N C D, B I) & (C D, N I) \\
(N C D, L I) & (C, N I) \\
(N C D, R I) & (S C, N I)
\end{array}
$$

$(N C D, N I)$

\footnotetext{
28 Theorem 1 also has a partial converse, which is of less importance for the sake of our argument; more information about this converse can be found in Lemma 7 and Remark 9 in the appendix.
} 
Proof It suffices to show that $\varphi$ and $\psi$ do not stand in any of the 9 other pairs:

- $\varphi$ and $\psi$ do not stand in $(C D, B I)$ :

For a reductio, suppose they $d o$ stand in those relations; since $\bullet(C D, B I)=(0,0,0,0)$ $=\perp$, it follows by Theorem 1 that $=\perp$, which contradicts the consistency of $\mathrm{S}$. Note that this case does not even rely on the contingency of $\varphi$ and $\psi$.

- $\varphi$ and $\psi$ do not stand in $(S C, L I)$ :

For a reductio, suppose they do stand in those relations; since $\bullet^{(S C, L I)}=(1,0,1,0)$, it follows by Theorem 1 that $\models \psi$, which contradicts the contingency of $\psi$. The cases $(S C, R I),(C, L I)$ and $(C, R I)$ yield the connectives $(1,1,0,0),(0,0,1,1)$ and $(0,1,0,1)$, respectively, and can thus be treated analogously.

- $\varphi$ and $\psi$ do not stand in $(C D, L I)$ :

For a reductio, suppose they do stand in those relations; since $\bullet(C D, L I)=(0,0,1,0)$, it follows by Theorem 1 that $\models \neg \varphi \wedge \psi$, and hence also $\models \neg \varphi$ and $\models \psi$, which contradict the contingency of both $\varphi$ and $\psi$. The cases $(S C, B I),(C D, L I)$ and $(C, B I)$ yield the connectives $(1,0,0,0),(0,0,1,0)$ and $(0,0,0,1)$, respectively, and can thus be treated analogously.

Theorems 1 and 2 connect the binary, truth-functional connectives on the one hand with pairs consisting of an opposition and an implication relation $(\mathscr{O} \mathscr{G} \times \mathscr{I} \mathscr{G})$ on the other. We finish this subsection by showing that this connection generalizes the connection between the original Aristotelian relations $(\mathscr{A} \mathscr{G})$ and their defining connectives, which was already hinted at by Bocheński (1959) and Williamson (1972).

Remark 5 Consider arbitrary contingent formulas $\varphi, \psi \in \mathscr{L}_{\mathrm{S}}$. If $C D(\varphi, \psi)$ or $C(\varphi, \psi)$ or $S C(\varphi, \psi)$, then it follows by Theorem 2 that $N I(\varphi, \psi)$. Similarly, if $S A(\varphi, \psi)$, i.e., $\operatorname{LI}(\varphi, \psi)$, then $N C D(\varphi, \psi)$. By Definition 6 , it holds that $\bullet(C D, N I)=(0,1,1,0)=$ $\underline{\vee}($ exclusive disjunction $), \bullet(C, N I)=(0,1,1,1)=\mid($ Sheffer's stroke $), \bullet(S C, N I)=(1,1,1,0)=$ $\vee$ and $\bullet^{(N C D, L I)}=(1,0,1,1)=\rightarrow$. Then by Theorem 1 it follows that:

- if $C D(\varphi, \psi)$, then $S \models \varphi \underline{\vee} \psi$, i.e., $\models \neg \Delta_{1}(\varphi, \psi)$ and $\models \neg \Delta_{4}(\varphi, \psi)$,

- if $C(\varphi, \psi)$, then $S \models \varphi \mid \psi$, i.e., $\models \neg \Delta_{1}(\varphi, \psi)$,

- if $\operatorname{SC}(\varphi, \psi)$, then $S \models \varphi \vee \psi$, i.e., $=\neg \Delta_{4}(\varphi, \psi)$,

- if $S A(\varphi, \psi)$, then $S \models \varphi \rightarrow \psi$, i.e., $=\neg \Delta_{2}(\varphi, \psi)$.

These entailments are entirely natural; after all, they merely express that the $\models$ parts of Definition 1 are necessary (but not sufficient) ${ }^{29}$ conditions for the Aristotelian relations. Bocheński (1959, p. 14) uses these entailments to define the Aristotelian relations, i.e., he views them as expressing necessary and sufficient conditions (e.g. $S C(\varphi, \psi): \Leftrightarrow \models \varphi \vee \psi$ ). In comparison to our Definition 1, Bocheński's definition can thus be seen as keeping the $\mid=$-conditions, while leaving out the $\not \models$ conditions. ${ }^{30}$ Obviously, both definitions are not equivalent; for example, although $S C(\varphi, \psi)$ entails that $S=\varphi \vee \psi$ according to both definitions, the converse is valid according to Bocheński's definition, but not according to our Definition 1 (cf. Remark 9 about the converse of Theorem 1 being only partial).

\footnotetext{
29 Of course, the $\models$ - and $\not \models$-parts together are sufficient.

30 Seuren (2010, p. 49) defines the Aristotelian relations in a similar way. Sanford (1968) compares the usual definition (cf. Definition 1) with that of Bocheński, and judges the former to be preferable.
} 


\section{Information in the Opposition and Implication Geometries}

This section is an investigation into the informativity of the opposition and implication geometries. Subsection 4.1 introduces the so-called 'information as range'perspective. Subsection 4.2 applies this perspective to the opposition and implication geometries, and Subsection 4.3 discusses some advantages of this application.

\subsection{Information as Range}

The 'information as range'-perspective on information is well-known in logic and formal semantics (Bar-Hillel and Carnap 1952; Hintikka 1970; Gamut 1991; Barwise 1997; Löbner 2002; Allo 2007; van Benthem and Martinez 2008; van Benthem 2011; Demey 2012b). We start by associating with each statement $\sigma$ a set $\mathbb{I}(\sigma)$, which is called the information range of $\sigma$, and whose elements are often referred to as 'states' or 'possible worlds'. The 'information as range'-perspective states that the informativity of a statement is inversely correlated with the size of its information range: "the more worlds there still are in the information range, the less information it contains" (Gamut 1991, p. 54). This inverse correlation is formally expressed by the definition of the informativity ordering $\leq_{i}$, which looks as follows:

$$
\sigma \leq_{i} \tau: \Leftrightarrow \mathbb{I}(\sigma) \supseteq \mathbb{I}(\tau) .
$$

Informally, this definition states that the statement $\tau$ is at least as informative as the statement $\sigma$ iff $\tau$ 's information range is a subset of $\sigma$ 's information range.

Since $\supseteq$ is a partial ordering (reflexive, transitive and antisymmetric), the informativity ordering $\leq_{i}$ is a preordering (reflexive and transitive). ${ }^{31}$ A strict informativity ordering $<_{i}$ can be defined by putting $\sigma<_{i} \tau: \Leftrightarrow\left(\sigma \leq_{i} \tau\right.$ and $\left.\tau \not \leq_{i} \sigma\right)$; this is a strict partial ordering (irreflexive and transitive) (Harel et al 2000, pp. 6-11).

In formal semantics, the information range of a statement $\sigma$ is usually identified with its truth set, i.e., the set of all possible worlds $w$ (in a given model $\mathbb{M}$ ) that make $\sigma$ true: $\mathbb{I}(\sigma)=[\sigma \sigma]_{\mathbb{M}}=\{w \in \mathbb{M}|w|=\sigma\}$. Consider the following example from Löbner (2002, pp. 64-66): let $\sigma$ be 'Donald Duck is a bird' and let $\tau$ be 'Donald Duck is a duck'. Since every possible world in which Donald Duck is a duck is also a world in which he is a bird, but not vice versa, it holds that $\mathbb{I}(\tau)=[[\tau] \subseteq[[\sigma]=\mathbb{I}(\sigma)$ and $\mathbb{I}(\sigma) \nsubseteq \mathbb{I}(\tau)$, and hence the 'information as range'-perspective states that $\sigma \leq_{i} \tau$ and $\tau \not \mathbb{L}_{i} \sigma$, respectively, and thus $\sigma<_{i} \tau$. This matches the semantic judgment that the nominal predicate 'is a duck' is strictly more informative than the nominal predicate 'is a bird'.

It should be emphasized that unlike other, more quantitatively oriented theories of information (Harremoës and Topsœ 2008), the 'information as range'-perspective does not yield any absolute informativity judgments (of the form ' $\sigma$ has informativity $k$ ', where $k \in[0,1]$ ), but only comparative informativity judgments (of the form ' $\sigma$

\footnotetext{
31 The information ordering $\leq_{i}$ is not antisymmetric, because from $\sigma \leq_{i} \tau$ and $\tau \leq_{i} \sigma$ it follows that the statements $\sigma$ and $\tau$ are equally informative (i.e., $\mathbb{I}(\sigma)=\mathbb{I}(\tau)$ ), but not that they are identical (i.e., not $\sigma=\tau)$.
} 
is at least as informative as $\tau$ ' and ' $\sigma$ is strictly more informative than $\tau^{\prime}$ ). For our current purposes, however, such comparative judgments will suffice.

\subsection{Information in the Opposition and Implication Geometries}

We will now show how the 'information as range'-perspective introduced above can be used to compare the informativity of opposition and implication relations. However, this perspective concerns the informativity of statements rather than relations. Therefore, it is first applied to statements of the form $R(\varphi, \psi)$, and subsequently this analysis is lifted from statements about relations to the relations themselves.

For any opposition or implication relation $R$ and formulas $\varphi, \psi \in \mathscr{L}_{\mathrm{S}}$, we consider the statement $R(\varphi, \psi)$, which says that $\varphi$ and $\psi$ stand in the relation $R$. This statement does not belong to the logic's object language $\left(\mathscr{L}_{S}\right)$, but rather to its metalanguage. Hence, it does not make sense to talk about $R(\varphi, \psi)$ being true in a given S-model $\mathbb{M}$; however, it does make sense to talk about $R(\varphi, \psi)$ being compatible with $\mathbb{M}$. Consequently, the information range of the statement $R(\varphi, \psi)$ does not consist of the models in which it is true, but rather of the models with which it is compatible.

Definition 7 Consider a relation $R \in \mathscr{O} \mathscr{G} \cup \mathscr{I} \mathscr{G}$ and formulas $\varphi, \psi \in \mathscr{L}_{\mathrm{S}}$. Let $\mathscr{C}_{\mathrm{S}}$ be the class of all models of $\mathrm{S}$. Then we define:

1. a model $\mathbb{M} \in \mathscr{C}_{S}$ is compatible with the statement $R(\varphi, \psi)$ iff

$$
\text { for } 1 \leq i \leq 4:\left(R(\varphi, \psi) \Rightarrow \mathrm{S} \models \neg \Delta_{i}(\varphi, \psi)\right) \Longrightarrow \mathbb{M} \models \neg \Delta_{i}(\varphi, \psi),
$$

2. the information range of the statement $R(\varphi, \psi)$ is

$$
\mathbb{I}(R(\varphi, \psi)):=\left\{\mathbb{M} \in \mathscr{C}_{\mathrm{S}} \mid \mathbb{M} \text { is compatible with } R(\varphi, \psi)\right\} .
$$

An S-model $\mathbb{M}$ is thus compatible with a statement $R(\varphi, \psi)$ iff it is not a countermodel to any of the universal claims in terms of which the truth of the statement $R(\varphi, \psi)$ is defined; in other words, iff all formulas $\neg \Delta_{i}(\varphi, \psi)$ that $R(\varphi, \psi)$ entails to be tautological (cf. Definitions 3 and 4) are satisfied by $\mathbb{M}$. Note that for most logical systems $\mathrm{S}$, the class $\mathscr{C}_{\mathrm{S}}$ of all S-models is a proper class, and thus the information ranges of statements $R(\varphi, \psi)$ will be proper classes too. This is not a problem for the 'information as range'-perspective, however, because this perspective only makes use of comparative statements: it makes perfect sense to say that $X \subseteq Y$ for proper classes $X$ and $Y$ (Jech 2002, p. 6).

Definition 7 provides a 'top-down' perspective on the information range of a statement $R(\varphi, \psi)$ : we start from the class of all models, and remove those that are not compatible with $R(\varphi, \psi)$. Lemma 4 provides an alternative, 'bottom-up' perspective, by characterizing information range as unions of truth classes, i.e., classes of models of the form $\llbracket\left[\Delta_{i}(\varphi, \psi) \rrbracket=\left\{\mathbb{M} \in \mathscr{C}_{\mathrm{S}} \mid \mathbb{M}=\Delta_{i}(\varphi, \psi)\right\}\right.$.

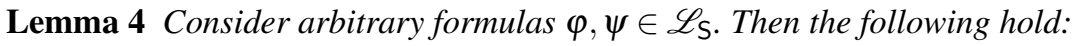




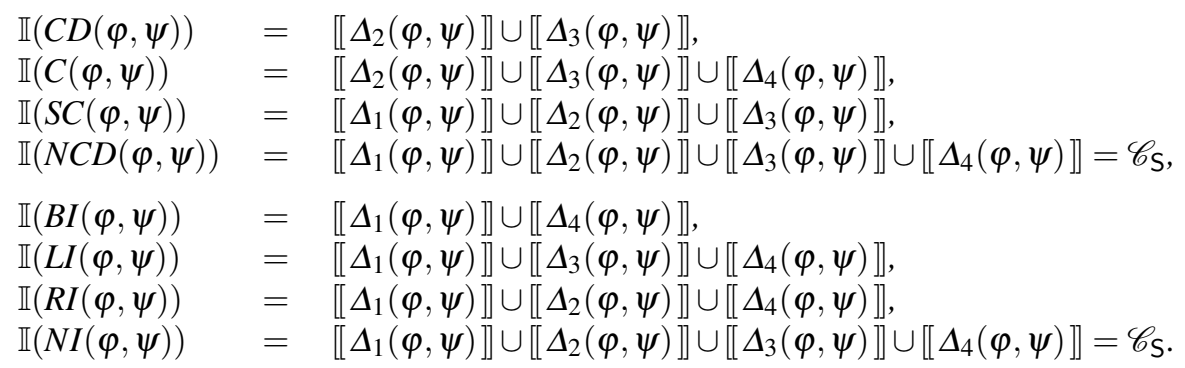

Proof We prove the first item. Recall that by Definition 3, $C D(\varphi, \psi)$ entails that $\mathrm{S} \models \neg \Delta_{i}(\varphi, \psi)$ for $i=1,4$, and hence, by Definition 7 , an $\mathrm{S}$-model $\mathbb{M}$ is compatible with $C D(\varphi, \psi)$ iff $\mathbb{M}=\neg \Delta_{1}(\varphi, \psi)$ and $\mathbb{M}=\neg \Delta_{4}(\varphi, \psi)(\dagger)$. Furthermore, note that it follows from the definitions of $\Delta_{i}$ that $\mathrm{S} \models \bigvee_{i=1}^{i=4} \Delta_{i}(\varphi, \psi)$ ( $\ddagger$ ). We thus get the following chain of identities:

$$
\begin{aligned}
\mathbb{I}(C D(\varphi, \psi)) & =\left\{\mathbb{M} \in \mathscr{C}_{S} \mid \mathbb{M} \text { is compatible with } C D(\varphi, \psi)\right\} \\
& =\left\{\mathbb{M} \in \mathscr{C}_{S} \mid \mathbb{M}=\neg \Delta_{1}(\varphi, \psi) \text { and } \mathbb{M}=\neg \Delta_{4}(\varphi, \psi)\right\} \\
& =\left\{\mathbb{M} \in \mathscr{C}_{S} \mid \mathbb{M}=\Delta_{2}(\varphi, \psi) \text { or } \mathbb{M}=\Delta_{3}(\varphi, \psi)\right\} \\
& \left.\left.=\llbracket \Delta_{2}(\varphi, \psi)\right] \cup \llbracket \Delta_{3}(\varphi, \psi)\right] .
\end{aligned}
$$

The other items are proved completely analogously.

We are now ready to move from the informativity of statements to that of relations. By universally quantifying over $\mathscr{L}_{\mathrm{S}}$, we lift the informativity ordering $\leq_{i}$ of statements of the form $R(\varphi, \psi)$ to an informativity ordering $\leq_{i}^{\forall}$ of the relations $R$ themselves. ${ }^{32}$

Definition 8 Consider relations $R, S \in \mathscr{O} \mathscr{G} \cup \mathscr{I} \mathscr{G}$. Then we define:

$$
R \leq_{i}^{\forall} S: \Leftrightarrow \forall \varphi \in \mathscr{L}_{\mathrm{S}}: \forall \psi \in \mathscr{L}_{\mathrm{S}}: R(\varphi, \psi) \leq_{i} S(\varphi, \psi) .
$$

Since $\leq_{i}$ is a preordering, its lifted version $\leq_{i}^{\forall}$ is a preordering as well. The strict version of this ordering is defined as follows:

$$
R<{ }_{i}^{\forall} S: \Leftrightarrow\left(R \leq_{i}^{\forall} S \text { and } S \mathbb{L}_{i}^{\forall} R\right)
$$

This is a strict partial ordering (Harel et al 2000, p. 11).

Definition 8 defines the informativity ordering $\leq_{i}^{\forall}$ for $\mathscr{O} \mathscr{G} \cup \mathscr{I} \mathscr{G}$, i.e., for opposition and implication relations collectively. Theorems 3 and 4 describe how $\leq_{i}^{\forall}$ orders the opposition and implication geometries separately. ${ }^{33}$

\footnotetext{
${ }^{32}$ Definition 8 involves a universal quantification over $\varphi$ and $\psi$, i.e., it makes use of a $\forall \forall$-pattern. One might wonder whether other quantification patterns could be used here. A promising candidate seems to be the $\exists \exists$-pattern: define $R \leq_{i}^{\exists} S$ iff $\exists \varphi \in \mathscr{L}_{S}: \exists \psi \in \mathscr{L}_{S}: R(\varphi, \psi) \leq_{i} S(\varphi, \psi)$. However, one can show that this $\leq_{i}^{\exists}$-ordering fails to make any distinctions within the two geometries, in the sense that for all $R, S \in \mathscr{O} \mathscr{G}$ it holds that $R \leq_{i}^{\exists} S$ (and similarly for $\mathscr{I} \mathscr{G}$ ). To see this, note that by applying Lemma 4 to the formulas $p$ and $\neg p$, we find for all $R, S \in \mathscr{O} \mathscr{G}$ that $\mathbb{I}(R(p, \neg p))=\mathscr{C}_{\mathrm{S}} \supseteq \mathscr{C}_{\mathrm{S}}=\mathbb{I}(S(p, \neg p))$, and thus $R(p, \neg p) \leq_{i} S(p, \neg p)$, from which it follows that $R \leq_{i}^{\exists} S$. Finally, one might consider the $\forall \exists$ - and $\exists \forall-$ patterns, but these asymmetrical patterns have even less intuive appeal. Thanks to an anonymous referee for suggesting us to explore this in more depth.

33 The only cross-geometry informativity statements that hold are $N C D \leq_{i}^{\forall} R$ and $N I \leq_{i}^{\forall} R$, for all relations $R \in \mathscr{O} \mathscr{G} \cup \mathscr{I} \mathscr{G}$. We will return to such cross-geometry statements in Subsection 5.1; in particular, see Definition 9.
} 
Theorem 3 The opposition geometry $\mathscr{O} \mathscr{G}$ is ordered by $\leq_{i}^{\forall}$ as follows:

- NCD $\leq_{i}^{\forall} C, N C D \leq_{i}^{\forall} S C, N C D \leq_{i}^{\forall} C D, C \leq_{i}^{\forall} C D$ and $S C \leq_{i}^{\forall} C D$,

- for all $R \in \mathscr{O} \mathscr{G}: R \leq_{i}^{\forall} R$,

- for all other pairs $(R, S) \in \mathscr{O} \mathscr{G}^{2}: R \not_{i}^{\forall} S$.

Proof We prove that $C \leq_{i}^{\forall} C D$ (the other items of the form $R \leq_{i}^{\forall} S$ are proved analogously). Consider arbitrary formulas $\varphi, \psi \in \mathscr{L}_{\mathrm{S}}$; it suffices to show that $C(\varphi, \psi) \leq_{i}$ $C D(\varphi, \psi)$. It follows from Lemma 4 that

$$
\begin{aligned}
\mathbb{I}(C D(\varphi, \psi)) & =\left[\left[\Delta_{2}(\varphi, \psi)\right] \cup\left[\left[\Delta_{3}(\varphi, \psi)\right]\right]\right. \\
& \subseteq\left[[ \Delta _ { 2 } ( \varphi , \psi ) ] \cup \left[[ \Delta _ { 3 } ( \varphi , \psi ) ] \cup \left[\left[\Delta_{4}(\varphi, \psi)\right]=\mathbb{I}(C(\varphi, \psi)) .\right.\right.\right.
\end{aligned}
$$

By the definition of the $\leq_{i}$, this means that $C(\varphi, \psi) \leq_{i} C D(\varphi, \psi)$.

We now prove that $C D \mathbb{Z}_{i}^{\forall} C$ (the other items of the form $R \not_{i}^{\forall} S$ are proved analogously). It suffices to show that $\exists \varphi, \psi \in \mathscr{L}_{C}: C D(\varphi, \psi) \mathbb{L}_{i} C(\varphi, \psi)$. Let $\varphi:=p$ and $\psi:=q$. Note that $\left[\left[\Delta_{4}(p, q)\right]\right]$ is non-empty (there certainly exists a model $\mathbb{M}$ such that $\left.\mathbb{M}=\Delta_{4}(p, q)\right)$; hence

$$
\begin{aligned}
\mathbb{I}(C(p, q)) & =\left[\left[\Delta_{2}(p, q)\right]\right] \cup\left[\left[\Delta_{3}(p, q)\right]\right] \cup\left[\left[\Delta_{4}(p, q)\right]\right] \\
& \nsubseteq\left[\left[\Delta_{2}(p, q)\right]\right] \cup\left[\left[\Delta_{3}(p, q)\right]\right]=\mathbb{I}(C D(\varphi, \psi)) .
\end{aligned}
$$

Again, by the definition of $\leq_{i}$, this means that $C D(p, q) \mathbb{L}_{i} C(p, q)$.

Theorem 4 The implication geometry $\mathscr{I} \mathscr{G}$ is ordered by $\leq_{i}^{\forall}$ as follows:

- NI $\leq_{i}^{\forall} L I, N I \leq_{i}^{\forall} R I, N I \leq_{i}^{\forall} B I, L I \leq_{i}^{\forall} B I$ and $R I \leq_{i}^{\forall} B I$,

- for all $R \in \mathscr{I} \mathscr{G}: R \leq_{i}^{\forall} R$,

- for all other pairs of relations $(R, S) \in \mathscr{I} \mathscr{G}^{2}: R \not_{i}^{\forall} S$.

Proof Completely analogous to the proof of Theorem 3.

It follows from Theorem 3 that $C \leq_{i}^{\forall} C D$ and $C D \not_{i}^{\forall} C$, and hence $C<_{i}^{\forall} C D$. Similarly, we get that $N C D<_{i}^{\forall} C, N C D<_{i}^{\forall} S C, N C D<_{i}^{\forall} C D$, and $S C<_{i}^{\forall} C D$. Furthermore, $C$ and $S C$ are $\leq_{i}$-incomparable. The opposition relations are thus ordered by informativity as in Figure 7(a). Completely analogously, it follows from Theorem 4 that the implication relations are ordered as in Figure 7(b). The relations $N C D$ and $N I$ are thus the least informative in their respective geometries, ${ }^{34}$ a property that will become crucial in Subsection 5.2.

\subsection{Motivating the Information Account}

In this subsection, we show that the application of the 'information as range'-perspective to the opposition and implication geometries is well-motivated. We first argue that it matches well with our intuitive informativity judgments, and then prove that it intertwines seamlessly with the internal and external structure of both geometries.

\footnotetext{
34 This is reflected in the code in Figure 5, which visualizes these two relations in grey instead of black (recall Footnote 20).
} 
Fig. 7 Informativity ordering of (a) the opposition geometry and (b) the implication geometry.

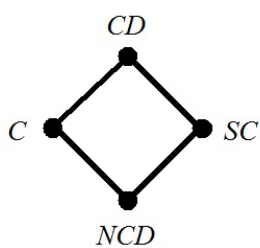

(a)

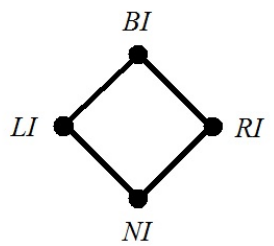

(b)

Intuitive Informativity Judgments. In the previous subsection, the 'information as range'-perspective was used to order the opposition and implication relations according to informativity. For example, it entails that $N C D$ is the least informative opposition relation, that $C D$ is the most informative opposition relation, and that $C$ and $S C$ are in between (cf. Theorem 3 and Figure 7). These theoretical claims seem to match our intuitive judgments about the relative informativity of the opposition relations.

We will describe a game to explain this. Recall that there are 16 binary, truthfunctional connectives. For each connective $\bullet$, we consider the formula $p \bullet q$; these 16 formulas form a Boolean algebra $\mathbb{B}_{4}$. We randomly choose a formula from $\mathbb{B}_{4}$, let's say $q$, and show it to the opponent $O p$. We then randomly select another formula $\varphi$ from $\mathbb{B}_{4}$ (possibly the one that was chosen before), but do not show it to $O p$. Instead, we determine the opposition relation that holds between $q$ and $\varphi$, and communicate this to $O p$. Based on this information, $O p$ has to guess the value of $\varphi$. There are four cases:

1. $C D(q, \varphi)$ : then $O p$ knows that $\varphi=\neg q$,

2. $C(q, \varphi)$ : then $O p$ knows that $\varphi \in\{p \wedge \neg q, \neg p \wedge \neg q, \perp\}$,

3. $S C(q, \varphi)$ : then $O p$ knows that $\varphi \in\{p \vee \neg q, \neg p \vee \neg q$, $\top\}$,

4. $N C D(q, \varphi)$ : then $O p$ knows that

$$
\varphi \in\{p, q, p \wedge q, \neg p, p \vee q, p \rightarrow q, p \leftrightarrow q, p \vee q, \neg p \wedge q\} .
$$

In the first case, $O p$ comes to know the exact value of $\varphi ; C D$ is thus the most informative opposition relation. In the second and third cases, $O p$ comes to know that $\varphi$ has one out of 3 values, but she remains uncertain as to which of these 3 ; hence, $C$ and $S C$ are strictly less informative than $C D$. In the fourth case, $O p$ merely comes to know that $\varphi$ has one out of 9 values; hence, $N C D$ is strictly less informative than $C$ and $S C$, and thus the least informative opposition relation.

One might object that this intuitive scenario only works because the formula $q$ sits in the middle level of $\mathbb{B}_{4}$, and that formulas in other levels will yield results that match less well with the informativity claims made by our account. ${ }^{35}$ For example, $q$ has the same number of contraries and subcontraries (viz., 3), while for formulas in other levels, this might not be the case. However, it should be noted that in general, the Boolean algebra $\mathbb{B}_{n}$ (with $n>1$ ) has levels $L_{0}, L_{1}, L_{2}, \ldots L_{n-2}, L_{n-1}, L_{n}$, and that every formula not belonging to $L_{0}, L_{1}, L_{n-1}$ or $L_{n}$ yields the right comparative

35 For the notion of 'level' in a Boolean algebra, or, more generally, in a poset, see Engel (1997, p. 7). 
results, i.e., the numbers of its contraries and of its subcontraries will be strictly between the number of its contradictories and the number of its noncontradictories. ${ }^{36}$ Furthermore, since $\left|L_{k}\right|=\left(\begin{array}{l}n \\ k\end{array}\right)=\frac{n !}{k !(n-k) !}$, it holds that

$$
\lim _{n \rightarrow \infty} \frac{\left|L_{0}\right|+\left|L_{1}\right|+\left|L_{n-1}\right|+\left|L_{n}\right|}{\left|\mathbb{B}_{n}\right|}=\lim _{n \rightarrow \infty} \frac{1+n+n+1}{2^{n}}=0 .
$$

Hence, the chance that a randomly chosen formula belongs to $L_{0}, L_{1}, L_{n-1}$ or $L_{n}$ (and thus yields results that do not entirely match with the informativity claims made by our account) vanishes for sufficiently large Boolean algebras.

The remarks made above apply not only to Boolean algebras of formulas, but to (finite) Boolean algebras in general, because the opposition and implication relations can be defined for any Boolean algebra $\mathbb{B}=\left\langle B, \wedge_{\mathbb{B}}, \vee_{\mathbb{B}}, \neg_{\mathbb{B}}, \perp_{\mathbb{B}}, \top_{\mathbb{B}}, \leq_{\mathbb{B}}\right\rangle$; for example, contrariety and left-implication in $\mathbb{B}$ are typically defined as follows (for any $x, y \in B):^{37}$

$$
\begin{array}{lllll}
C_{\mathbb{B}}(x, y) & \text { iff } & x \wedge_{\mathbb{B}} y=\perp_{\mathbb{B}} & \text { and } & x \vee_{\mathbb{B}} y \neq \top_{\mathbb{B}}, \\
L I_{\mathbb{B}}(x, y) & \text { iff } & x \leq_{\mathbb{B}} y & \text { and } & y \mathbb{Z}_{\mathbb{B}} x .
\end{array}
$$

Coherence with the Structure of the Geometries. A major theoretical advantage of the informativity perspective on the opposition and implication geometries is that it intertwines seamlessly with the internal and external structure of these geometries, which was described in Subsection 3.3.

The informativity ordering of the opposition geometry is fully described by Theorem 3, and visualized by Figure 7(a). It is immediately clear from this figure that from the informativity perspective, $C$ and $S C$ play symmetrical roles: both are strictly in between $N C D$ and $C D$. Formally, this can be expressed as follows:

$$
N C D<_{i}^{\forall} C<_{i}^{\forall} C D \text { and } N C D<_{i}^{\forall} S C<_{i}^{\forall} C D \text {. }
$$

However, there is a theoretical redundancy here, since each of these two series of inequalities actually follows from the other one. Using the mapping N12 that was defined in Corollary 2, this can be reformulated as follows:

Lemma 5 For all relations $R, S \in \mathscr{O} \mathscr{G}: R \leq_{i}^{\forall} S$ iff $N 12(R) \leq_{i}^{\forall} N 12(S)$.

Proof For all $R, S \in \mathscr{O} \mathscr{G}$, it holds that

\footnotetext{
36 More precisely, a formula in level $L_{i}$ has 1 contradictory, $2^{n-i}-1$ contraries, $2^{i}-1$ subcontraries, and $\left(2^{n-i}-1\right)\left(2^{i}-1\right)$ noncontradictories. Note that $1<\left\{2^{n-i}-1,2^{i}-1\right\}<\left(2^{n-i}-1\right)\left(2^{i}-1\right)$ iff $1<i<n-1$, i.e., a formula yields the right comparative results iff it does not belong to $L_{0}, L_{1}, L_{n-1}$ or $L_{n}$. Finally, note that if $i \approx \frac{n}{2}$, then $2^{n-i}-1 \approx 2^{i}-1$, i.e., formulas sitting (approximately) in the middle level of $\mathbb{B}_{n}$ indeed have (approximately) the same number of contraries and subcontraries.

37 The opposition relations are thus typically defined in terms of $\wedge_{\mathbb{B}}$ and $\vee_{\mathbb{B}}$, while the implication relations are typically defined in terms of $\leq_{\mathbb{B}}$. This suggests that the distinction between the opposition and implication geometries is analogous to the distinction between the algebraic and order-theoretic perspectives on Boolean algebras (Davey and Priestley 2002, pp. 33-41). Furthermore, it is well-known that both perspectives are equivalent to each other (via $x \leq \mathbb{B} y \Leftrightarrow x \wedge_{\mathbb{B}} y=x$ ); this is analogous to the connection between the opposition and implication geometries described in Lemma 3.
} 


$$
\begin{aligned}
R \leq_{i}^{\forall} S & \Leftrightarrow \quad \forall \varphi, \psi \in \mathscr{L}_{S}: R(\varphi, \psi) \leq_{i} S(\varphi, \psi) \\
& \Leftrightarrow \quad \forall \varphi, \psi \in \mathscr{L}_{S}: N 12(R)(\neg \varphi, \neg \psi) \leq_{i} N 12(S)(\neg \varphi, \neg \psi) \\
& \Leftrightarrow \quad \forall \varphi, \psi \in \mathscr{L}_{S}: N 12(R)(\varphi, \psi) \leq_{i} N 12(S)(\varphi, \psi) \\
& \Leftrightarrow \quad N 12(R) \leq_{i}^{\forall} N 12(S) .
\end{aligned}
$$

The $\dagger$-labeled equivalence holds because of Corollary 2 . The $\ddagger$-labeled equivalence holds because of the universal quantification over $\mathscr{L}_{\mathrm{S}}$, and because it holds for any opposition relation $R$ and formulas $\varphi, \psi$ that $R(\varphi, \psi)$ iff $R(\neg \neg \varphi, \neg \neg \psi)$.

Similar remarks can be made about the connection between the informativity ordering of the implication geometry-as described by Theorem 4 and visualized by Figure 7 (b) - and the internal structure of this geometry (if $\mathscr{O} \mathscr{G}$ is replaced with $\mathscr{I} \mathscr{G}$ in Lemma 5, the proof remains valid).

We now turn to the connection between the informativity perspective and the geometries' external structure (i.e., how they are related to each other). It is immediately clear from Figure 7 that $\mathscr{O} \mathscr{G}$ and $\mathscr{I} \mathscr{G}$ are ordered in exactly the same way. Formally, this can be expressed as follows:

$$
N C D<_{i}^{\forall}\{C, S C\}<_{i}^{\forall} C D \text { and } N I<_{i}^{\forall}\{L I, R I\}<_{i}^{\forall} B I .
$$

However, there is a theoretical redundancy here, since each of these two series of inequalities actually follows from the other one. Using the mapping $N 2$ that was defined in Corollary 3, this can be reformulated as follows:

Lemma 6 For all relations $R, S \in \mathscr{O} \mathscr{G} \cup \mathscr{I} \mathscr{G}: R \leq_{i}^{\forall} S$ iff $N 2(R) \leq_{i}^{\forall} N 2(S)$.

Proof Completely analogous to the proof of Lemma 5, but based on Corollary 3 instead of Corollary 2.

Summing up, there are certain facts about the informativity ordering of the opposition and implication geometries that can be obtained in two distinct ways:

1. by deriving them directly from the 'information as range'-perspective on these geometries (Definitions 7 and 8); this was done in Theorems 3 and 4;

2 . by combining that application with the geometries' internal and external structure (Corollaries 2 and 3); this was done in Lemmas 5 and 6.

These considerations can be seen as evidence for the theoretical robustness of the informativity account that was described in this section.

\section{Information in the Aristotelian Geometry and its Diagrams}

In the previous two sections we have introduced the opposition and implication geometries, and shown how the 'information as range'-perspective can be applied to them. This conceptual machinery will now be used to explicate the intuition that the Aristotelian square is highly informative. This will be done in two successive steps: in Subsection 5.1 we will show that the Aristotelian geometry is an informative geometry, and in Subsection 5.2 we will show that within this geometry, the well-known square is a highly informative diagram. Finally, in Subsection 5.3 we will reassess the purported problems of the Aristotelian geometry in the light of these informativity considerations. 


\subsection{Information in the Aristotelian Geometry}

The Aristotelian geometry (Definition 1) can be characterized as being hybrid between the opposition geometry (Definition 3 ) and the implication geometry (Definition 4): it consists of three opposition relations $(C D, C$ and $S C$ ) and one implication relation $(L I$, i.e., $S A)$. From an informativity perspective, the former three are the most informative relations in the opposition geometry, while the latter is second most informative in the implication geometry (Figure 7). Hence, the Aristotelian geometry is hybrid in an informationally optimal way.

One might object that for the Aristotelian geometry to be truly informationally optimal, it would have to include $B I$, since that implication relation is strictly more informative than $L I$. Additionally, since $R I$ is second most informative too, it seems arbitrary to include $L I$ and not $R I$. We will now provide a more formal account of the hybrid nature of the Aristotelian geometry, which adequately addresses both these objections, and thus supports the conclusion regarding its informational optimality.

For our purposes, it will be necessary to compare informativity 'across geometries'. For example, considering Figure 7, there is a clear sense in which $C D$ is strictly more informative in $\mathscr{O} \mathscr{G}$ than $R I$ is in $\mathscr{I} \mathscr{G}: C D$ is the most informative relation in $\mathscr{O} \mathscr{G}$, while $R I$ is only amongst the second most informative relations in $\mathscr{I} \mathscr{G}$. Still, one can check that it does not hold that $R I<_{i}^{\forall} C D .{ }^{38}$ However, the mapping $N 2$ defined in Corollary 3 does enable us to make such cross-geometrical informativity comparisons (this is justified because informativity is invariant under this mapping; cf. Lemma 6). For example, although it does not hold that $R I{ }_{i}^{\forall} C D$, it does hold that $N 2(R I)=S C<_{i}^{\forall} C D$, and therefore $C D$ will be called the 'winner' of $\{C D, R I\}$.

Definition 9 Consider arbitrary relations $R \in \mathscr{O} \mathscr{G}$ and $S \in \mathscr{I} \mathscr{G}$. Then the winner of $\{R, S\}$ is defined as follows:

- $S$ is the winner iff $R<{ }_{i}^{\forall} N 2(S)$;

$-R$ is the winner iff $N 2(S)<_{i}^{\forall} R$.

For example, $B I$ is the winner of $\{C, B I\}$, since $C<_{i}^{\forall} C D=N 2(B I)$. Furthermore, $\{C, R I\}$ does not yield a winner at all: $C \nless_{i}^{\forall} S C=N 2(R I)$, so $R I$ is not the winner; and $N 2(R I)=S C \nless_{i}^{\forall} C$, so $C$ is not the winner either.

We now establish two theorems which capture the informational optimality of the Aristotelian geometry. Theorem 5 below states that all Aristotelian relations (between contingent formulas) are winners, i.e., informationally optimal.

Theorem 5 Consider arbitrary contingent formulas $\varphi, \psi \in \mathscr{L}_{\mathrm{S}}$. Let $R \in \mathscr{O} \mathscr{G}$ and $S \in \mathscr{I} \mathscr{G}$ be the unique relations such that $R(\varphi, \psi)$ and $S(\varphi, \psi)$, respectively.

1. If $R \in \mathscr{A} \mathscr{G}$, then $R$ is the winner of $\{R, S\}$.

2. If $S \in \mathscr{A} \mathscr{G}$, then $S$ is the winner of $\{R, S\}$.

Proof First, suppose that $R \in \mathscr{A} \mathscr{G}$. Since $\varphi$ and $\psi$ are contingent, it follows by Theorem 2 that $R \in\{C D, C, S C\}$ and $S=N I$, and hence $R$ is the winner of $\{R, S\}$. Second, suppose that $S \in \mathscr{A} \mathscr{G}$. Since $\varphi$ and $\psi$ are contingent, it follows by Theorem 2 that $R=N C D$ and $S=L I$, and hence $S$ is the winner of $\{R, S\}$.

38 Theorems 3 and 4 apply only 'locally' to $\mathscr{O} \mathscr{G}$ and $\mathscr{I} \mathscr{G}$, respectively; cf. Footnote 33. 
Since Theorem 5 states that all Aristotelian relations are winners, it is natural to ask the converse question: are all winners Aristotelian? Theorem 6 states that this is indeed by and large the case.

Theorem 6 Let $\varphi, \psi \in \mathscr{L}_{\mathrm{S}}$ and $R \in \mathscr{O} \mathscr{G}, S \in \mathscr{I} \mathscr{G}$ be as in Theorem 5 .

1. If $R$ is the winner of $\{R, S\}$, then $R \in \mathscr{A} \mathscr{G}$.

2. If $S$ is the winner of $\{R, S\}$, then $S \in \mathscr{A} \mathscr{G} \cup\{B I, R I\}$.

Proof First, suppose that $R$ is the winner of $\{R, S\}$. Since $\varphi$ and $\psi$ are contingent, it follows by Theorem 2 that $R \in\{C D, C, S C\} \subseteq \mathscr{A} \mathscr{G}$. Second, suppose that $S$ is the winner of $\{R, S\}$. Since $\varphi$ and $\psi$ are contingent, it follows by Theorem 2 that $S \in\{B I, L I, R I\} \subseteq \mathscr{A} \mathscr{G} \cup\{B I, R I\}$.

The two cases where the winner is $B I$ or $R I$ (and thus does not belong to $\mathscr{A} \mathscr{G}$ ) correspond exactly to the two objections against the informational optimality of $\mathscr{A} \mathscr{G}$ that were raised at the beginning of this subsection. We will now discuss how these cases are resolved in the class of Aristotelian diagrams. ${ }^{39}$

First, note that by definition, Aristotelian diagrams are semantic entities, i.e., they do not contain any distinct equivalent formulas (Definition 2). Logical equivalence coincides with $B I$, and therefore, in any diagram, $B I$ holds exactly between each formula and itself. ${ }^{40}$ The $B I$-relations thus need not be visualized explicitly in the Aristotelian diagrams: their place is predetermined by their definition and does not vary from diagram to diagram (they occur exactly as the 'loops' between each formula and itself).

Second, note that if $\operatorname{RI}(\varphi, \psi)$, then by Theorem $2 N C D(\varphi, \psi)$. By Lemma 1 it follows that $N C D(\psi, \varphi)$ and $L I(\psi, \varphi)$. The winner of $\{N C D, L I\}$ is $L I$, which does belong to $\mathscr{A} \mathscr{G}$. Therefore, the $R I$-relations need not be visualized explicitly in the Aristotelian diagrams: they correlate exactly with the $L I$-relations (because $L I$ and $R I$ offer two complementary perspectives on truth propagation; cf. Footnote 21). ${ }^{41}$

The remarks above suggest that from an information visualization viewpoint, $B I$ and $R I$ are redundant (even though they are among the more informative implication relations). After all, their place in the Aristotelian diagrams is predictable, either absolutely (the BI-relations occur exactly as the 'loops'), or on the basis of the other Aristotelian relations (the $R I$-relations occur exactly wherever there are $L I$-relations

\footnotetext{
39 Since this discussion applies to all Aristotelian diagrams, it rightly belongs in this subsection. The next subsection, in contrast, will distinguish between various particular Aristotelian diagrams, e.g. the concrete square, the concrete Sesmat-Blanché hexagon, etc.

40 This argument is made fully precise in Definitions 11 and 12 and Lemma 8 in the appendix.

41 From a theoretical perspective, the case of $L I / R I$ described above (which is based on the equivalence $L I(\varphi, \psi) \Leftrightarrow R I(\psi, \varphi)$; cf. Lemma 1$)$ seems to be exactly similar to the case of $C / S C$ (which is based on the equivalence $C(\varphi, \psi) \Leftrightarrow S C(\neg \varphi, \neg \psi)$; cf. Lemma 2) and to that of $C / L I$ (which is based on the equivalence $C(\varphi, \psi) \Leftrightarrow L I(\varphi, \neg \psi)$; cf. Lemma 3). This might suggest that $S C$ and $L I$ can unproblematically be left out of the Aristotelian diagrams as well. From a visual perspective, however, the latter two cases are entirely different from the first. The $L I / R I$ case does not require considering formulas other than $\varphi$ and $\psi$; hence, in the diagrams, the $R I$ relations occur in exactly the same place as the original $L I$ relations (but in the reverse direction). On the other hand, the $C / S C$ and $C / L I$ cases require considering formulas other than $\varphi$ and $\psi$, viz., ( $\neg \varphi$ and) $\neg \psi$; hence, in the diagrams, the $S C$ and $L I$ relations occur in other places than the original $C$ relations.
} 
in the converse direction). It is important to emphasize, however, that this visual redundancy of $B I$ and $R I$ is perfectly compatible with their importance in the internal and external structure of the implication geometry (cf. Subsection 3.3).

Example 1 Consider the fragment $\{\square p, \diamond p, \square \neg p\}$. Parts (a), (b) and (c) of Figure 8 show the respective opposition, Aristotelian and implication diagrams for this fragment (using the code of Figure 5). ${ }^{42}$

Fig. 8 The (a) opposition, (b) Aristotelian and (c) implication diagram for $\{\square p, \diamond p, \square \neg p\}$

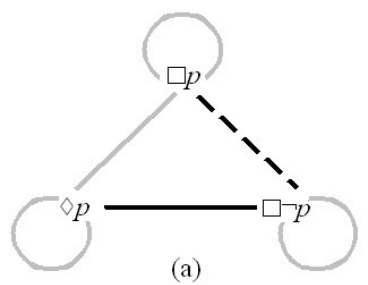

(a)

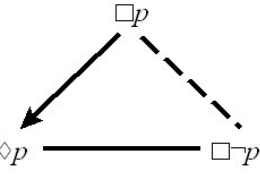

(b)

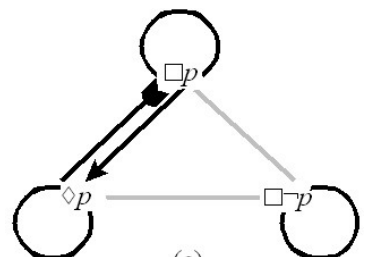

(c)

For each pair of distinct formulas, the Aristotelian diagram contains the winner of the corresponding opposition and implication relations:

- $C(\square p, \square \neg p)$ and $N I(\square p, \square \neg p)$ : the winner of $\{C, N I\}$ is $C \in \mathscr{A} \mathscr{G}$;

- $C D(\diamond p, \square \neg p)$ and $N I(\diamond p, \square \neg p)$ : the winner of $\{C D, N I\}$ is $C D \in \mathscr{A} \mathscr{G}$;

- NCD $(\square p, \diamond p)$ and $L I(\square p, \diamond p)$ : the winner of $\{N C D, L I\}$ is $L I \in \mathscr{A} \mathscr{G}$.

Furthermore, note that for each formula $\varphi$ in this fragment, it holds that $N C D(\varphi, \varphi)$ and $B I(\varphi, \varphi)$. Although $B I$ is the winner of $\{N C D, B I\}$, it is not visualized in the Aristotelian diagram, because of the reasons stated above. Finally, note that $N C D(\diamond p, \square p)$ and $R I(\diamond p, \square p)$; although $R I$ is the winner of $\{N C D, R I\}$, it is not visualized in the Aristotelian diagram (but its converse $L I$ is visualized; cf. the third item above).

Summing up, Theorems 5 and 6 together state that a relation between contingent formulas is a winner if and only if it is Aristotelian (modulo $B I$ and $R I$ ). Hence, each Aristotelian diagram offers an informationally optimal picture of its vertices: all winners are represented in the Aristotelian diagram (modulo $B I$ and $R I$ ), and all Aristotelian relations are winners, i.e., contrapositively, all non-winners are not represented in the Aristotelian diagram.

\subsection{Information in the Aristotelian Diagrams}

In the previous subsection we have argued that the Aristotelian geometry is informationally optimal in a positive sense: a relation between contingent formulas is Aristotelian if and only if it is a winner (i.e., cross-geometrically most informative). In

\footnotetext{
42 Note that Figure 8 shows the same three diagrams as Figure 6, but in a different order: we will henceforth put the Aristotelian diagram in between the opposition and implication diagrams, to reflect $\mathscr{A} \mathscr{G}$ being hybrid between $\mathscr{O} \mathscr{G}$ and $\mathscr{I} \mathscr{G}$.
} 
this subsection we will focus on a negative aspect of this informational optimality, by showing that Aristotelian diagrams avoid (the combination of) the least informative relations as much as possible. It turns out that this minimally informative combination does not occur in certain diagrams, but is unavoidable in others.

One question that was left unanswered in the previous subsection is: what if $\{R, S\}$ does not yield a winner at all? Such cases certainly exist; cf. the $\{C, R I\}$ example below Definition 9. However, in the case of contingent formulas, if $\{R, S\}$ does not yield a winner, it follows by contraposition on Theorem 5 that neither $R$ nor $S$ is an Aristotelian relation. Moreover, the following theorem states that in this case, $R$ and $S$ are uniquely identified.

Theorem 7 Let $\varphi, \psi \in \mathscr{L}_{S}$ and $R \in \mathscr{O} \mathscr{G}, S \in \mathscr{I} \mathscr{G}$ be as in Theorem 5. If neither $R$ nor $S$ is the winner of $\{R, S\}$, then $R=N C D$ and $S=N I$.

Proof Since $R$ is not the winner of $\{R, S\}$, it follows by Theorem 2 that $R \notin\{C D, C, S C\}$. Since $S$ is not the winner of $\{R, S\}$, it follows by Theorem 2 that $S \notin\{B I, L I, R I\}$. It follows by Theorem 2 that $(R, S)=(N C D, N I)$.

The combination of $N C D$ and $N I$ effectively occurs; for example, it is easy to check that $p$ and $\diamond p \wedge \diamond \neg p$ are both non-contradictory and in non-implication. This combination of relations will be crucial to the remainder of this subsection, and is therefore given a separate name, viz., 'unconnectedness'. ${ }^{43}$

Definition 10 Let $\varphi, \psi \in \mathscr{L}_{S}$ be arbitrary formulas. Then $\varphi$ and $\psi$ are said to be unconnected, written $U(\varphi, \psi)$, iff they are in non-contradiction and non-implication. Formally: $U(\varphi, \psi): \Leftrightarrow N C D(\varphi, \psi)$ and $N I(\varphi, \psi)$.

The term 'unconnectedness' suggests that unconnected formulas stand in no Aristotelian relation at all. ${ }^{44}$ The following theorem shows that this is essentially correct (and thus justifies the term 'unconnectedness'). ${ }^{45}$

Theorem 8 Consider formulas $\varphi, \psi$ in an arbitrary Aristotelian diagram. Then:

$\varphi$ and $\psi$ do not stand in any Aristotelian relation $\Leftrightarrow \varphi$ and $\psi$ are unconnected.

Proof Let $R \in \mathscr{O} \mathscr{G}$ and $S \in \mathscr{I} \mathscr{G}$ be the unique relations such that $R(\varphi, \psi)$ and $S(\varphi, \psi)$, respectively. For the right-to-left direction, note that if $\varphi$ and $\psi$ are unconnected, then $R=N C D \notin \mathscr{A} \mathscr{G}$ and $S=N I \notin \mathscr{A} \mathscr{G}$. We now prove the left-to-right direction. Assume that $R \notin \mathscr{A} \mathscr{G}$ and $S \notin \mathscr{A} \mathscr{G}$. Since $\varphi$ and $\psi$ belong to an Aristotelian diagram, they are (by Definition 2) contingent and non-equivalent. Hence

\footnotetext{
43 Although its combinatorial and informational properties have not been systematically explored so far, the notion of unconnectedness as such has surfaced at various places in the literature, usually under the label 'logical independence'; for example, see Hughes (1987, p. 99), Sion (1996, p. 36), Béziau (2003, p. 226), Karger (2003, p. 435), Seuren (2010, p. 50), Campos-Benítez (2012, pp. 101ff.), Jacquette (2012, p. 86) and Read (2012b, p. 104).

44 For example, Campos-Benítez states that "independent sentences [...] are not contrary neither subcontrary nor contradictory or subaltern: they have no relationship at all" (2012, p. 103).

45 In other words, Theorem 8 characterizes the absence of any Aristotelian relation in a positive way, viz., as the joint presence of an opposition and an implication relation.
} 
$S \neq B I$. Furthermore, note that if $S=R I$, then (by Lemma 1) $L I(\psi, \varphi)$, and since $L I$ is an Aristotelian relation, this contradicts the assumption that $\varphi$ and $\psi$ do not stand in any Aristotelian relation; therefore, $S \neq R I$. Hence, by contraposition on Theorem 6 we get that neither $R$ nor $S$ is the winner of $\{R, S\}$. By Theorem 7 it follows that $R=N C D$ and $S=N I$, i.e., $\varphi$ and $\psi$ are unconnected.

Remark 6 The proofs of Theorems 5-8 all make essential use of Theorem 2. The 7 pairs of opposition and implication relations singled out by that theorem are structured according to informativity: they are exactly the pairs that consist of (i) the least informative relation in either the opposition or the implication geometry ( $N C D$ or $N I$, respectively) and (ii) any of the four relations in the other geometry. Formally, this means that the set of those 7 pairs can be written as

$$
(\{N C D\} \times \mathscr{I} \mathscr{G}) \cup(\mathscr{O} \mathscr{G} \times\{N I\}) .
$$

Of course, in this way the unconnectedness combination gets 'counted twice', since $(N C D, N I) \in(\{N C D\} \times \mathscr{I} \mathscr{G}) \cap(\mathscr{O} \mathscr{G} \times\{N I\})$. The importance of these 7 pairs was already noted by John Buridan (Karger 2003), although he did not view them as pairs of more primitive notions, nor in the light of informativity considerations as we have done here.

Unconnectedness is thus the combination of the two relations that are least informative in their respective geometries. ${ }^{46}$ The Aristotelian diagrams avoid this minimally informative combination as much as possible. This can be seen as the negative counterpart of the informativity claim argued for in the previous subsection, viz., that the Aristotelian diagrams consist entirely and exclusively of winners. These negative and positive theses jointly constitute the informational optimality of the Aristotelian diagrams.

Obviously, whether or not unconnectedness occurs in a given Aristotelian diagram is fully determined by whether there are unconnected formulas amongst its vertices. Considering the diagrams mentioned in Section 2, it turns out that some of them have unconnectedness, while others do not. We consider them one by one. ${ }^{47}$

First, consider the usual Aristotelian square for the fragment $\mathscr{F}_{4}=\{\square p, \square \neg p, \diamond p, \diamond \neg p\}$, which was already displayed in Figure 2(b). Note that there is no unconnectedness in this fragment: for all $\varphi, \psi$, it holds that if $\operatorname{NCD}(\varphi, \psi)$ then not $N I(\varphi, \psi)$ (or equivalently, if $N I(\varphi, \psi)$ then not $N C D(\varphi, \psi))$, and hence, there are no $\varphi, \psi$ such that $\operatorname{NCD}(\varphi, \psi)$ and $N I(\varphi, \psi)$ simultaneously, i.e., such that $U(\varphi, \psi)$. Visually speaking, each grey $N C D$ relation in the opposition square in Figure 9(a) corresponds to a black $(L I / R I / B I)$ relation in the implication square in Figure 9(c), and vice versa, each grey $N I$ relation on the right corresponds to a black $(C D / C / S C)$ relation on the

\footnotetext{
46 This might explain why some authors, while acknowledging the existence of this relation, deny its logical relevance. For example, according to Seuren, unconnectedness is "a legitimate relation between L-propositions producing truth under certain conditions, yet [...] plays no role [...] in any logic" (2010, p. 50). Similarly, Béziau thinks that by treating unconnectedness as a 'real' logical relation, "we are going too far and confusing here negation with distinction" (2003, p. 226).

47 These diagrams fit into an exhaustive typology that is currently being developed (Smessaert and Demey 2014). This typology includes several other types of Aristotelian diagrams, which have various proportions of unconnectedness among their relations.
} 
left. Therefore, the Aristotelian square in Figure 9(b) contains no unconnectedness: each pair of distinct formulas stands in some Aristotelian relation.

Fig. 9 The (a) opposition, (b) Aristotelian and (c) implication square for $\{\square p, \square \neg p, \diamond p, \diamond \neg p\}$

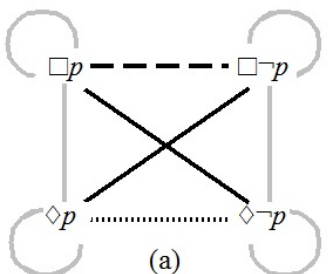

(a)

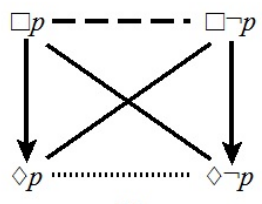

(b)

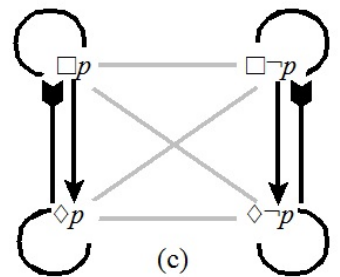

(c)

Next, we consider two types of Aristotelian hexagons: the Sesmat-Blanché hexagon for the fragment $\mathscr{F}_{6 a}=\mathscr{F}_{4} \cup\{\square p \vee \square \neg p, \diamond p \wedge \diamond \neg p\}$ and the Sherwood-Czeżowski hexagon for the fragment $\mathscr{F}_{6 b}=\mathscr{F}_{4} \cup\{p, \neg p\}$, which were already displayed in Figure 3(a) and (b), respectively. Neither of these fragments contains any unconnectedness (in spite of their significant Boolean differences; cf. Smessaert 2012a), so each pair of distinct formulas in the Aristotelian hexagons in Figures 10(b) and 11(b) stands in some Aristotelian relation.

Fig. 10 The (a) opposition, (b) Aristotelian and (c) implication Sesmat-Blanché hexagon for $\{\square p, \square \neg p, \diamond p, \diamond \neg p, \square p \vee \square \neg p, \diamond p \wedge \diamond \neg p\}$

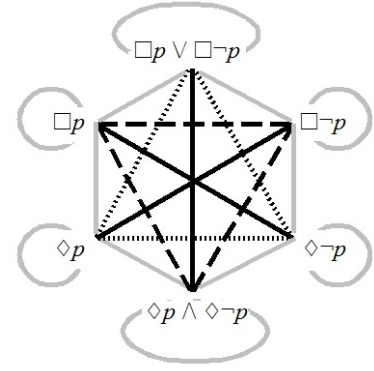

(a)

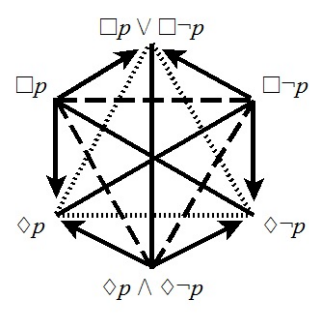

(b)

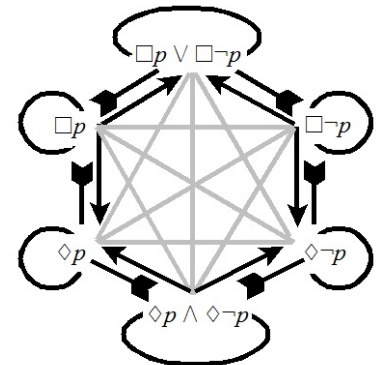

(c)

None of the Aristotelian diagrams considered thus far contains unconnectedness. This changes, however, when we turn to the Béziau octagon for the fragment $\mathscr{F}_{8}=$ $\mathscr{F}_{6 a} \cup \mathscr{F}_{6 b}$, cf. Figure 3(c) above (despite the fact that it is the 'sum' of the hexagons for $\mathscr{F}_{6 a}$ and $\mathscr{F}_{6 b}$, which themselves do not contain any unconnectedness). For example, we have $N C D(p, \diamond p \wedge \diamond \neg p)$ and $N I(p, \diamond p \wedge \diamond \neg p)$, and thus $U(p, \diamond p \wedge \diamond \neg p)$. Similarly, it holds that $U(p, \square p \vee \square \neg p), U(\neg p, \diamond p \wedge \diamond \neg p)$ and $U(\neg p, \square p \vee \square \neg p)$. Visually speaking, these four formulas are thus connected by four grey NCD relations in the opposition octagon in Figure 12(a) and by four grey NI relations in the implication octagon in Figure 12(c); hence, they are not connected by any relation in the Aristotelian octagon in Figure 12(b). 
Fig. 11 The (a) opposition, (b) Aristotelian and (c) implication Sherwood-Czeżowski hexagon for $\{\square p, \square \neg p, \diamond p, \diamond \neg p, p, \neg p\}$

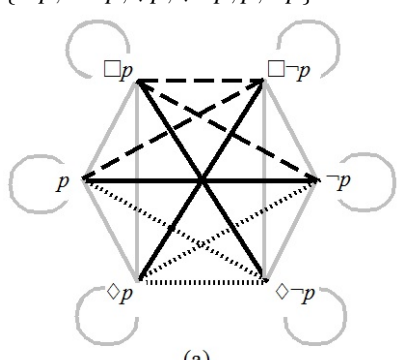

(a)

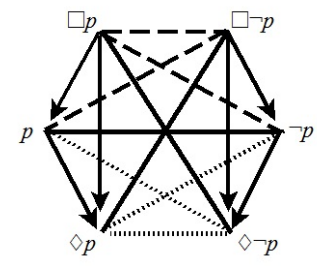

(b)

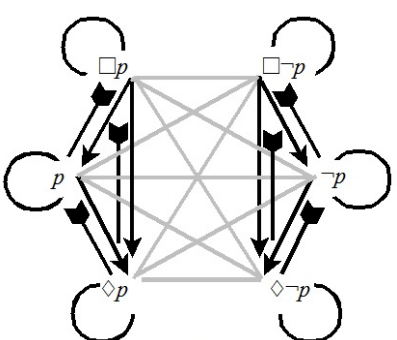

(c)

Fig. 12 The (a) opposition, (b) Aristotelian and (c) implication Béziau octagon for $\{\square p, \square \neg p, \diamond p, \diamond \neg p, \square p \vee \neg \square p, \diamond p \wedge \diamond \neg p, p, \neg p\}$ (the $R I$ relations have not been visualized in (c) for the sake of visual clarity)

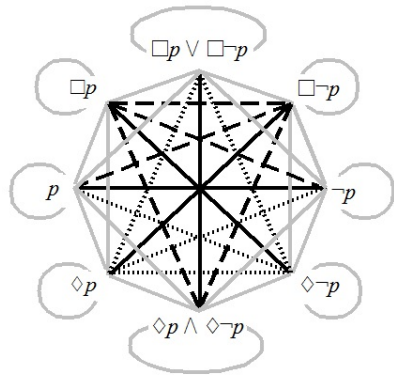

(a)

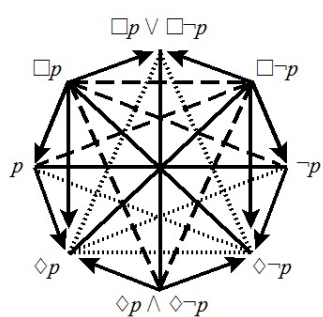

(b)

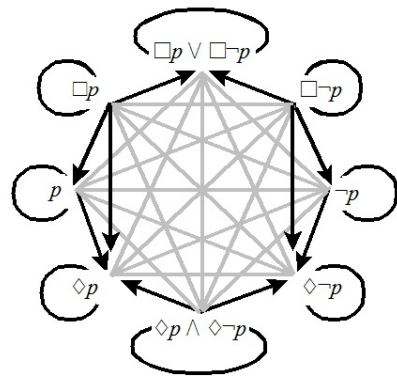

(c)

To summarize, the Aristotelian square and hexagons discussed above do not contain unconnectedness, and thus avoid the combination of the least informative opposition and implication relations. However, this combination does occur in the Béziau octagon for $\mathscr{F}_{8}$, and thus also in every Aristotelian diagram that contains this octagon as a subdiagram (such as the rhombic dodecahedron; cf. Smessaert 2009).

It does not hold in general, however, that larger diagrams contain more unconnectedness. To see this, note that there exist still other 'large' Aristotelian diagrams which do not contain any unconnectedness (such as the cube in Moretti (2009a), which consists of 8 formulas). Conversely, there also exist 'small' Aristotelian diagrams which do contain unconnectedness. Consider, for example, the three squares for the fragment $\mathscr{F}_{4}^{\prime}=\{\square p \vee \square \neg p, \diamond p \wedge \diamond \neg p, p, \neg p\}{ }^{48}$ The outer edges of the opposition and implication squares in Figure 13(a) and (c) are grey NCD and NI relations, respectively. Hence, there are no Aristotelian relations at the outer edges of the Aristotelian square in Figure 13(b), which thus degenerates into "an X of opposition" (Béziau and Payette 2012, p. 13). This abundance of unconnectedness might explain why such degenerate diagrams have been rarely studied in the literature.

\footnotetext{
48 Note that $\mathscr{F}_{4}^{\prime}=\mathscr{F}_{8}-\mathscr{F}_{4}$, i.e., the squares in Figure 13 can be seen as the result of 'subtracting' the classical squares in Figure 9 from the corresponding Béziau octagons in Figure 12.
} 
Fig. 13 The (a) opposition, (b) Aristotelian and (c) implication square for $\{\square p \vee \neg \square p, \diamond p \wedge \diamond \neg p, p, \neg p\}$

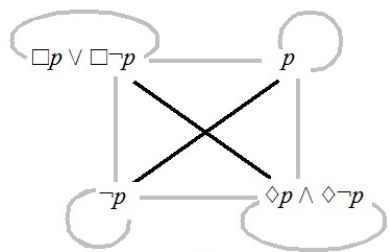

(a)

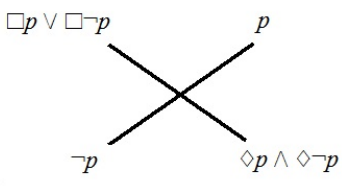

(b)

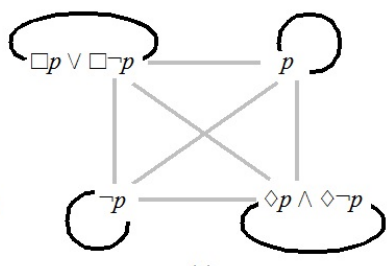

(c)

\subsection{Reassessing the Problems of the Aristotelian Geometry}

In Subsection 3.1 we discussed three problems of the Aristotelian geometry. We will now show that the informativity perspective developed in this section sheds new light on these issues: rather than being 'brute facts' about the Aristotelian geometry, they can be seen as necessary consequences of its informational optimality.

The first problem was that the Aristotelian relations are not mutually exclusive; for example, we have both $C(p \wedge \neg p, p)$ and $L I(p \wedge \neg p, p)$. Recall that any two formulas $\varphi$ and $\psi$ stand in exactly one opposition relation and exactly one implication relation; hence, if $\varphi$ an $\psi$ stand in two distinct Aristotelian relations $R$ and $S$ at all, then one of those relations ( say $R$ ) will be an opposition relation, and the other one (say $S$ ) an implication relation. Note that $R$ and $S$ cannot be both the winner of $\{R, S\}$, since otherwise we would have $R<_{i}^{\forall} N 2(S)<_{i}^{\forall} R$, which contradicts the transitivity and irreflexivity of $<_{i}^{\forall}$. Hence, if $\varphi$ and $\psi$ are contingent, then contraposition on Theorem 5 yields that $R$ and $S$ cannot be both Aristotelian. In other words, $\varphi$ and $\psi$ can stand in two distinct Aristotelian relations only if at least one of them is noncontingent. This fact was already known (recall Footnote 16); what we discussed here is how it arises out of the informational interplay between the opposition and implication geometries.

The second problem was that the Aristotelian relations are not jointly exhaustive; for example, $p$ and $\diamond p \wedge \diamond \neg p$ stand in no Aristotelian relation whatsoever. Recall that unconnectedness is the combination of the least informative opposition and implication relations $(N C D$ and $N I)$. Theorem 8 states that two formulas stand in no Aristotelian relation if and only if they are unconnected. In other words, the Aristotelian geometry is indeed not exhaustive, but only inasmuch as this is required by its informational optimality. This means, in particular, that there are no 'fortuitous' failures of exhaustiveness: if two formulas stand in no Aristotelian relation, this can only be because they stand in the least informative opposition and implication relations. ${ }^{49}$

The third and final problem was that the Aristotelian geometry is conceptually confused, because it consists of opposition as well as implication relations. Recall that (modulo the cases of $B I$ and $R I$ ) Theorem 6 states that all winners are Aris-

\footnotetext{
49 Note that the second problem involves contingent formulas (such as $p$ and $\diamond p \wedge \diamond \neg p$ ), and thus occurs both in the Aristotelian geometry and its diagrams. In contrast, we showed above that non-contingency is a necessary condition for the first problem, which thus never occurs in the diagrams (since these contain only contingent formulas).
} 
totelian. Since opposition as well as implication relations can be winners, it follows that both kinds of relations belong to the Aristotelian geometry, which thus ends up being hybrid between the opposition and implication geometries. Furthermore, Theorem 5 states that a relation is Aristotelian only if it is a winner; in other words, the Aristotelian geometry includes no more relations than is required by informativity considerations.

\section{Conclusion}

In this paper, we have argued that the classical Aristotelian square of oppositions is highly informative. After distinguishing between the Aristotelian geometry and its concrete diagrams, we introduced two more logical geometries: the opposition and implication geometries. This is a well-motivated move: the new geometries are highly structured (Lemmas 1-3) and have a canonical correspondence with the binary, truthfunctional connectives (Theorem 1). We then extended the well-known 'information as range'-perspective from statements to logical relations, thus obtaining an informativity ordering on the opposition and implication geometries (Theorems 3-4). This ordering is highly intuitive, and matches well with the geometries' structural properties (Lemmas 5-6). We then argued that the Aristotelian geometry is hybrid between the opposition and implication geometries in an informationally optimal way, since it consists entirely and exclusively of winners (Theorems 5-6). Finally, we studied the notion of unconnectedness (Theorems 7-8) and found that this minimally informative combination does not occur in the classical Aristotelian square, but does appear in some of its extensions (such as the Béziau octagon).

The following question now arises: what about diagrams such as the SesmatBlanché and Sherwood-Czeżowski hexagons? After all, these diagrams are as highly informative as the classical square (they are also Aristotelian diagrams that do not contain any unconnectedness), yet they are much less widely known than the square. In other words, aren't these hexagons counterexamples to our explanation of the square's success in terms of its informativity?

Answering this question requires the introduction of one more logical geometry, viz., the duality geometry. This geometry is concerned with (the interplay of) internal and external negations on an operator (e.g. $\diamond=\neg \square \neg$ ), and is well-known in linguistics (van Benthem 1991; Löbner 1989; Löbner 1990; Westerståhl 2012) and logic (Demey 2012a; Libert 2012; Veloso et al 2011). Although the duality geometry is sometimes confused with the Aristotelian geometry (D'Alfonso 2012; Mélès 2012), they are conceptually independent of each other (Löbner 1990; Smessaert 2012b; Westerståhl 2012). It turns out that if the duality geometry is taken into account as well, then the classical square is singled out as the most informative diagram (being strictly more informative than all of its extensions, including the hexagons). The details of this additional move will be spelled out in a follow-up paper.

In future research, we intend to explore the various connections between the informational account developed here and the exhaustive typology of Aristotelian diagrams developed in Smessaert and Demey (2014). For example, in this typology we often make use of bitstrings (an algebraic representation of the formulas), and some 
of the informational notions defined here are directly related to bitstring properties (such as length, i.e., number of bit positions). It can be shown, for example, that two formulas are unconnected only if their bitstring representations have a length of at least 4 bit positions.

Acknowledgements Earlier versions of this paper were presented at Trends in Logic XI (Bochum, June 3-5 2012) and CLMPS 14 (Nancy, July 19-26 2011); we would like to thank the audiences of these talks for their helpful remarks and suggestions. We would also like to thank Dany Jaspers, Alessio Moretti, Fabien Schang, Margaux Smets and three anonymous referees for their extensive feedback on earlier versions of this paper. The second author is financially supported by a $\mathrm{PhD}$ fellowship of the Research FoundationFlanders (FWO).

\section{Appendix}

Remark 7 The group $\mathbf{G}_{\mathbf{4}}=\langle\{I d, F, N 12, F N 12\}, \circ\rangle$ is isomorphic to the Klein fourgroup $\mathbf{V}$. The latter has generators $x, y$ and can be presented as $\langle x, y| x^{2}=1, y^{2}=$ $1, x y=y x\rangle$. A concrete isomorphism $\imath: \mathbf{V} \rightarrow \mathbf{G}_{\mathbf{4}}$ is determined by where it sends the generators of $\mathbf{V}: \imath(x)=F$ and $\imath(y)=N 12$. The Cayley table of $\mathbf{G}_{\mathbf{4}}$ thus looks as follows:

\begin{tabular}{c|cccc}
$\circ$ & $I d$ & $F$ & $N 12$ & $F N 12$ \\
\hline$I d$ & $I d$ & $F$ & $N 12$ & $F N 12$ \\
$F$ & $F$ & $I d$ & $F N 12$ & $N 12$ \\
$N 12$ & $N 12$ & $F N 12$ & $I d$ & $F$ \\
$F N 12$ & $F N 12$ & $N 12$ & $F$ & $I d$
\end{tabular}

This group acts faithfully on $\mathscr{G}$, and partitions it into six $\mathbf{G}_{\mathbf{4}}$-orbits:
1) $\mathbf{G}_{\mathbf{4}}(C D)=\{C D\}$,
2) $\mathbf{G}_{\mathbf{4}}(C)=\mathbf{G}_{\mathbf{4}}(S C)=\{C, S C\}$
4) $\mathbf{G}_{\mathbf{4}}(B I)=\{B I\}$,
3) $\mathbf{G}_{\mathbf{4}}(N C D)=\{N C D\}$,
5) $\mathbf{G}_{\mathbf{4}}(L I)=\mathbf{G}_{\mathbf{4}}(R I)=\{L I, R I\}$,
6) $\mathbf{G}_{\mathbf{4}}(N I)=\{N I\}$.

Remark 8 The group $\mathbf{G}_{\mathbf{8}}=\langle\{I d, N 1, N 2, N 12, F, F N 1, F N 2, F N 12\}, \circ\rangle$ is isomorphic to the dihedral group of order 8 , i.e., $\mathbf{D}_{8}$. The latter has generators $x, y$ and can be presented as $\left\langle x, y \mid x^{4}=1, y^{2}=1, y x y x=1\right\rangle$. A concrete isomorphism $\imath: \mathbf{D}_{\mathbf{8}} \rightarrow \mathbf{G}_{\mathbf{8}}$ is determined by where it sends the generators of $\mathbf{D}_{\mathbf{8}}: \imath(x)=F N 2$ and $\imath(y)=F$. The Cayley table of $\mathbf{G}_{\mathbf{8}}$ thus looks as follows:

\begin{tabular}{c|cccccccc}
$\circ$ & $I d$ & $N 1$ & $N 2$ & $N 12$ & $F$ & $F N 1$ & $F N 2$ & $F N 12$ \\
\hline$I d$ & $I d$ & $N 1$ & $N 2$ & $N 12$ & $F$ & $F N 1$ & $F N 2$ & $F N 12$ \\
$N 1$ & $N 1$ & $I d$ & $N 12$ & $N 2$ & $F N 2$ & $F N 12$ & $F$ & $F N$ \\
$N 2$ & $N 2$ & $N 12$ & $I d$ & $N 1$ & $F N 1$ & $F$ & $F N 12$ & $F N 2$ \\
$N 12$ & $N 12$ & $N 2$ & $N 1$ & $I d$ & $F N 12$ & $F N 2$ & $F N 1$ & $F$ \\
$F$ & $F$ & $F N 1$ & $F N 2$ & $F N 12$ & $I d$ & $N 1$ & $N 2$ & $N 12$ \\
$F N 1$ & $F N 1$ & $F$ & $F N 12$ & $F N 2$ & $N 2$ & $N 12$ & $I d$ & $N 1$ \\
$F N 2$ & $F N 2$ & $F N 12$ & $F$ & $F N 1$ & $N 1$ & $I d$ & $N 12$ & $N 2$ \\
$F N 12$ & $F N 12$ & $F N 2$ & $F N 1$ & $F$ & $N 12$ & $N 2$ & $N 1$ & $I d$
\end{tabular}

This group acts faithfully on $\mathscr{G}$, and partitions it into three $\mathbf{G}_{\mathbf{8}}$-orbits: 
1) $\mathbf{G}_{8}(C D)=\mathbf{G}_{8}(B I)=\{C D, B I\}$,

2) $\mathbf{G}_{8}(C)=\mathbf{G}_{8}(S C)=\mathbf{G}_{8}(L I)=\mathbf{G}_{8}(R I)=\{C, S C, L I, R I\}$,

3) $\mathbf{G}_{8}(N C D)=\mathbf{G}_{8}(N I)=\{N C D, N I\}$.

Lemma 7 Consider a binary, truth-functional connective $\bullet$. Then for all formulas $\varphi, \psi \in \mathscr{L}_{\mathrm{S}}$ such that $\mathrm{S}=\varphi \bullet \psi$, the following holds:

$$
\text { for all } 1 \leq i \leq 4 \text { : if } \bullet_{i}=0 \text { then } \mathrm{S} \models \neg \Delta_{i}(\varphi, \psi) \text {. }
$$

Proof Suppose that $\bullet_{i}=0$. By definition of the propositional function $\Delta_{i}$, this means that $\mathrm{S} \models \Delta_{i}(\varphi, \psi) \rightarrow \neg(\varphi \bullet \psi)$. Since $\mathrm{S} \models \varphi \bullet \psi$, it follows that $\mathrm{S} \models \neg \Delta_{i}(\varphi, \psi)$.

Remark 9 Lemma 7 can be seen as a partial converse of Theorem 1. To see this more clearly, recall Remark 1 about the opposition and implication geometries being defined in terms of $\Delta_{1}-\Delta_{4}$. Theorem 1 moves from an opposition relation and an implication relation, i.e., $\Delta_{1}-\Delta_{4}$, to a binary connective. Lemma 7 goes exactly in the other direction: it moves from a binary connective to $\Delta_{1}-\Delta_{4}$.

Of course, Lemma 7 is only a partial converse of Theorem 1, because it states that $\mathrm{S}=\neg \Delta_{i}(\varphi, \psi)$ if $i=0$, but remains silent about the case $i=1$. Based on Definition 6, one might expect that $\mathrm{S} \not \models \neg \Delta_{i}(\varphi, \psi)$ if $i=1$, but this does not hold in general. Consider, for example, the binary connective $\vee=(1,1,1,0)$ and the formulas $p$ and $\neg p$ from classical propositional logic (CPL). Since CPL $\models p \vee \neg p$ and $\vee_{1}=1$, one would erroneously conclude that CPL $\forall \neg \neg \Delta_{1}(p, \neg p)$, i.e., CPL $\forall \neg(p \wedge \neg p)$.

Definition 11 Let $S$ be a logical system as in Definition 1 . Recall that $\mathscr{G}_{S}=\mathscr{O} \mathscr{G}_{S} \cup$ $\mathscr{I} \mathscr{G}_{S}$ is the set of all opposition and implication relations for $S$. The pair $\mathbb{A}_{S}:=$ $\left\langle\mathscr{L}_{\mathrm{S}}, \mathscr{G}_{\mathrm{S}}\right\rangle$ is thus a relational structure, in the sense of Dunn and Hardegree (2001). Note that $\mathrm{S}$ has a notion of logical equivalence $\equiv_{\mathrm{S}} \subseteq \mathscr{L}_{\mathrm{S}} \times \mathscr{L}_{\mathrm{S}}$, defined by $\varphi \equiv_{\mathrm{S}} \psi: \Leftrightarrow$ $\mathrm{S} \models \varphi \leftrightarrow \psi$. The equivalence class of $\varphi \in \mathscr{L}_{\mathrm{S}}$ is defined as $[\varphi]_{\equiv_{\mathrm{S}}}:=\left\{\psi \in \mathscr{L}_{\mathrm{S}} \mid \varphi \equiv_{\mathrm{S}}\right.$ $\psi\}$. This equivalence relation is actually even a congruence relation on $\mathbb{A}_{S}$ (Dunn and Hardegree 2001, Definition 2.6.2). In the following definition and lemma, the subscript $S$ will be left implicit.

Definition 12 Given the relational structure $\mathbb{A}=\langle\mathscr{L}, \mathscr{G}\rangle$ and the congruence relation $\equiv$ on $\mathbb{A}$, we define the quotient structure $\mathbb{A} / \equiv:=\langle\mathscr{L}|\equiv, \mathscr{G}| \equiv\rangle$, with $\mathscr{L} \mid \equiv:=$ $\{[\varphi] \mid \varphi \in \mathscr{L}\}$, and each relation $R / \equiv \in \mathscr{G} / \equiv$ defined as follows: $([\varphi],[\psi]) \in R / \equiv$ $: \Leftrightarrow \exists \psi^{\prime} \in[\psi]:\left(\varphi, \psi^{\prime}\right) \in R$ (Dunn and Hardegree 2001, p. 23).

Lemma 8 Since $B I \in \mathscr{G}$, the quotient structure $\mathbb{A} / \equiv$ will also contain the relation $B I / \equiv$. But since $\equiv$ and $B I$ are actually the same relation, $B I / \equiv$ is the identity relation on $\mathscr{L} / \equiv$.

Proof For any $[\varphi],[\psi] \in \mathscr{L} / \equiv$, we have:

$$
\begin{aligned}
([\varphi],[\psi]) \in B I / \equiv & \Leftrightarrow \exists \psi^{\prime} \in[\psi]:\left(\varphi, \psi^{\prime}\right) \in B I \\
& \Leftrightarrow \exists \psi^{\prime} \in \mathscr{L}: \psi \equiv \psi^{\prime} \text { and } \varphi \equiv \psi^{\prime} \\
& \Leftrightarrow \varphi \equiv \psi \\
& \Leftrightarrow[\varphi]=[\psi] .
\end{aligned}
$$

Hence, each $[\varphi]$ stands in the $B I / \equiv$-relation to exactly one element, viz., itself. 


\section{References}

Allo P (2007) Logical pluralism and semantic information. Journal of Philosophical Logic 36:659-694 van der Auwera J (1996) Modality: The three-layered scalar square. Journal of Semantics 13:181-195 Bar-Hillel Y, Carnap R (1952) An outline of a theory of semantic information. Tech. Rep. 247, MIT, Cambridge, MA

Barwise J (1997) Information and impossibilities. Notre Dame Journal of Formal Logic 38:488-515

van Benthem J (1991) Linguistic universals in logical semantics. In: Zaefferer D (ed) Semantic Universals and Universal Semantics, Groningen-Amsterdam Studies in Semantics, vol 12, Foris, Berlin, pp $17-$ 36

van Benthem J (2011) Logical Dynamics of Information and Interaction. Cambridge University Press, Cambridge

van Benthem J, Martinez M (2008) The stories of logic and information. In: Adriaans P, van Benthem J (eds) Philosophy of Information, Elsevier, pp 217-280

Béziau JY (2003) New light on the square of oppositions and its nameless corner. Logical Investigations 10:218-232

Béziau JY, Payette G (2012) Preface. In: Béziau JY, Payette G (eds) The Square of Opposition. A General Framework for Cognition, Peter Lang, pp 9-22

Blanché R (1952) Quantity, modality, and other kindred systems of categories. Mind 61:369-375

Blanché R (1966) Structures Intellectuelles. Essai sur l'organisation systématique des concepts. Librairie Philosophique J. Vrin, Paris

Bocheński JM (1959) A Precis of Mathematical Logic. Reidel, Dordrecht

Campos-Benítez JM (2012) The medieval modal octagon and the S5 Lewis modal system. In: Béziau JY, Jacquette D (eds) The Square of Opposition. A General Framework for Cognition, Springer, Basel, pp 99-116

Carnap R (1947) Meaning and Necessity. A Study in Semantics and Modal Logic. University of Chicago Press, Chicago, IL

Carnielli W, Pizzi C (2008) Modalities and Multimodalities. Springer, Berlin

Chatti S (2012) Logical oppositions in Arabic logic: Avicenna and Averroes. In: Béziau JY, Jacquette D (eds) Around and Beyond the Square of Opposition, Springer, Basel, pp 21-40

Chatti S, Schang F (2013) The cube, the square and the problem of existential import. History and Philosophy of Logic 32:101-132

Correia M (2012) Boethius on the square of opposition. In: Béziau JY, Jacquette D (eds) Around and Beyond the Square of Opposition, Springer, Basel, pp 41-52

Czeżowski T (1955) On certain peculiarities of singular propositions. Mind 64:392-395

D’Alfonso D (2012) The square of opposition and generalized quantifiers. In: Béziau JY, Jacquette D (eds) Around and Beyond the Square of Opposition, Springer, Basel, pp 219-227

Davey BA, Priestley HA (2002) Introduction to Lattices and Order (Second Edition). Cambridge University Press, Cambridge

Demey L (2012a) Algebraic aspects of duality diagrams. In: Cox PT, Plimmer B, Rodgers P (eds) Diagrammatic Representation and Inference, Lecture Notes in Computer Science, vol 7352, Springer, Berlin, pp 300-302

Demey L (2012b) Narrative and information: Comment on Löwe. In: Allo P, Primiero G (eds) Proceedings of the Third Workshop in the Philosophy of Information, KVAB, Brussels, pp 29-34

Demey L (2012c) Structures of oppositions for public announcement logic. In: Béziau JY, Jacquette D (eds) Around and Beyond the Square of Opposition, Springer, Basel, pp 313-339

Demey L, Smessaert H (2014) The relationship between Aristotelian and Hasse diagrams. In: Dwyer T, Purchase H, Delaney A (eds) Diagrammatic Representation and Inference, Lecture Notes in Computer Science, vol 8578, Springer, Berlin, pp 215-229

Dubois D, Prade H (2012) From Blanché's hexagonal organization of concepts to formal concept analysis and possibility theory. Logica Universalis 6:149-169

Dunn JM, Hardegree GM (2001) Algebraic Methods in Philosophical Logic. Oxford University Press, Oxford

Enderton H (2001) A Mathematical Introduction to Logic (Second Edition). Academic Press, San Diego, $\mathrm{CA}$

Engel K (1997) Sperner Theory. Cambridge University Press, Cambridge

Fitting M, Mendelsohn RL (1998) First-Order Modal Logic. Kluwer, Dordrecht 
Gamut LTF (1991) Logic, Language, and Meaning. Volume 1: Introduction to Logic. University of Chicago Press, Chicago, IL

Gombocz W (1990) Apuleius is better still: a correction to the square of opposition. Phronesis 43:124-131

Harel D, Kozen D, Tiuryn J (2000) Dynamic Logic. MIT Press, Cambridge, MA

Harremoës P, TopsœF (2008) The quantitative theory of information. In: Adriaans P, van Benthem J (eds) Philosophy of Information, Elsevier, pp 171-216

Hintikka J (1970) On semantic information. In: Hintikka J, Suppes P (eds) Information and Inference, Reidel, Dordrecht, pp 3-27

Horn LR (1989) A Natural History of Negation. University of Chicago Press, Chicago, IL

Horn LR (1990) Hamburgers and truth: Why Gricean explanation is Gricean. In: Hall K (ed) Proceedings of the Sixteenth Annual Meeting of the Berkeley Linguistics Society, Berkeley Linguistics Society, Berkeley, pp 454-471

Horn LR (2010) Contradiction. In: Zalta EN (ed) Stanford Encyclopedia of Philosophy (Summer 2012 edition), CSLI

Horn LR (2012) Histoire d'*O: Lexical pragmatics and the geometry of opposition. In: Béziau JY, Payette G (eds) The Square of Opposition. A General Framework for Cognition, Peter Lang, pp 393-426

Hughes GE (1987) The modal logic of John Buridan. In: Corsi G, Mangione C, Mugnai M (eds) Atti del convegno internazionale di storia della logica, le teorie delle modalità, CLUEB, pp 93-111

Jacoby P (1950) A triangle of opposites for types of propositions in Aristotelian logic. New Scholasticism $24: 32-56$

Jacoby P (1960) Contrariety and the triangle of opposites in valid inferences. New Scholasticism 34:141169

Jacquette D (2012) Thinking outside the square of opposition box. In: Béziau JY, Jacquette D (eds) Around and Beyond the Square of Opposition, Springer, Basel, pp 73-92

Jaspers D (2012) Logic and colour. Logica Universalis 6:227-248

Jech T (2002) Set Theory (Third Millenium Edition). Springer, Berlin

Karger E (2003) John Buridan's theory of the logical relations between general modal formulae. In: Braakhuis HAG, Kneepkens CH (eds) Aristotle's Peri Hermeneias in the Later Middle Ages, Ingenium, Groningen-Haren, pp 429-444

Kauffman LH (2001) The mathematics of Charles Sanders Peirce. Cybernetics \& Human Knowing 8:79_ 110

Khomskii Y (2012) William of Sherwood, singular propositions and the hexagon of opposition. In: Béziau JY, Payette G (eds) The Square of Opposition. A General Framework for Cognition, Peter Lang, Bern, pp 43-60

Koslow A (1992) A Structuralist Theory of Logic. Cambridge University Press, Cambridge

Koslow A (1999) The implicational nature of logic: A structural account. European Review of Philosophy 4:111-155

Kretzmann N (1966) William of Sherwood's Introduction to Logic. University of Minnesota Press, Minneapolis, $\mathrm{MN}$

Lenzen W (2012) How to square knowledge and belief. In: Béziau JY, Jacquette D (eds) Around and Beyond the Square of Opposition, Springer, Basel, pp 305-311

Libert T (2012) Hypercubes of duality. In: Béziau JY, Jacquette D (eds) Around and Beyond the Square of Opposition, Springer, Basel, pp 293-301

Löbner S (1989) German schon - erst - noch: an integrated analysis. Linguistics and Philosophy 12:167212

Löbner S (1990) Wahr neben Falsch. Duale Operatoren als die Quantoren natürlicher Sprache. Max Niemeyer Verlag, Tübingen

Löbner S (2002) Understanding Semantics. Hodder Arnold, London

Londey D, Johanson C (1984) Apuleius and the square of opposition. Phronesis 29:165-173

Luzeaux D, Sallantin J, Dartnell C (2008) Logical extensions of Aristotle's square. Logica Universalis 2:167-187

McNamara P (2010) Deontic logic. In: Zalta EN (ed) Stanford Encyclopedia of Philosophy (Summer 2012 edition), CSLI

Mélès B (2012) No group of opposition for constructive logic: The intuitionistic and linear cases. In: Béziau JY, Jacquette D (eds) Around and Beyond the Square of Opposition, Springer, Basel, pp 201217

Moretti A (2009a) The geometry of logical opposition. PhD thesis, University of Neuchâtel

Moretti A (2009b) The geometry of standard deontic logic. Logica Universalis 3:19-57 
Moretti A (2012a) From the "logical square" to the "logical poly-simplexes". A quick survey of what happened in between. In: Béziau JY, Payette G (eds) The Square of Opposition. A General Framework for Cognition, Peter Lang, Bern, pp 119-156

Moretti A (2012b) Why the logical hexagon? Logica Universalis 6:69-107

Parry WT, Hacker EE (1991) Aristotelian Logic. State University of New York Press, Albany, NY

Parsons T (2006) The traditional square of opposition. In: Zalta EN (ed) Stanford Encyclopedia of Philosophy (Summer 2012 edition), CSLI

Peckhaus V (2012) Algebra of logic, quantification theory, and the square of opposition. In: Béziau JY, Payette G (eds) The Square of Opposition. A General Framework for Cognition, Peter Lang, Bern, pp $25-41$

Read S (2012a) Aristotle and Lukasiewicz on existential import, available online at www.standrews.ac.uk/ slr/Existential_Import.pdf

Read S (2012b) John Buridan's theory of consequence and his octagons of opposition. In: Béziau JY, Jacquette D (eds) Around and Beyond the Square of Opposition, Springer, Basel, pp 93-110

Rini AA, Cresswell MJ (2012) The World-Time Parallel. Tense and Modality in Logic and Metaphysics. Cambridge University Press, Cambridge

Rotman JJ (1995) An Introduction to the Theory of Groups (Fourth Edition). Springer, New York, NY

Sanford DH (1968) Contraries and subcontraries. Noûs 2:95-96

Sauriol P (1968) Remarques sur la théorie de l'hexagone logique de Blanché. Dialogue 7:374—390

Schang F (2012a) Abstract logic of opposition. Logic and Logical Philosophy 21:415-438

Schang F (2012b) Oppositions and opposites. In: Béziau JY, Jacquette D (eds) Around and Beyond the Square of Opposition, Springer, Basel, pp 147-173

Schang F (2012c) Questions and answers about oppositions. In: Béziau JY, Payette G (eds) The Square of Opposition. A General Framework for Cognition, Peter Lang, Bern, pp 289-320

Schang F (2013) Logic in opposition. Studia Humana 2:31-45

Sesmat A (1951) Logique II. Les Raisonnements. La syllogistique. Hermann, Paris

Seuren P (2010) The Logic of Language. Language from Within, volume II. Oxford University Press, Oxford

Seuren P (2012a) Does a leaking O-corner save the square? In: Béziau JY, Jacquette D (eds) Around and Beyond the Square of Opposition, Springer, Basel, pp 129-138

Seuren P (2012b) From logical intuitions to natural logic. In: Béziau JY, Payette G (eds) The Square of Opposition. A General Framework for Cognition, Peter Lang, Bern, pp 231-288

Sion A (1996) Future Logic. Lulu.com, Geneva

Smessaert H (2009) On the 3D visualisation of logical relations. Logica Universalis 3:303-332

Smessaert H (2012a) Boolean differences between two hexagonal extensions of the logical square of oppositions. In: Cox PT, Plimmer B, Rodgers P (eds) Diagrammatic Representation and Inference, Lecture Notes in Computer Science, vol 7352, Springer, Berlin, pp 193-199

Smessaert H (2012b) The classical Aristotelian hexagon versus the modern duality hexagon. Logica Universalis 6:171-199

Smessaert H, Demey L (2014) The logical geometry of the Aristotelian rhombic dodecahedron, ms.

Uckelman S (2009) Anselm's logic of agency. Logical Analysis and History of Philosophy 12:248-268

Veloso SRM, Veloso PAS, Veloso PP (2011) A tool for analysing logics. Electronic Notes in Theoretical Computer Science 269:125-137

Westerståhl D (2012) Classical vs. modern squares of opposition, and beyond. In: Béziau JY, Payette G (eds) The Square of Opposition. A General Framework for Cognition, Peter Lang, Basel, pp 195-229

Williamson C (1972) Squares of opposition: Comparisons between syllogistic and propositional logic. Notre Dame Journal of Formal Logic 13:497-500

Zellweger S (1997) Untapped potential in Peirce's iconic notation for the sixteen binary connectives. In: Houser N, Roberts DD, Van Evra J (eds) Studies in the Logic of Charles Peirce, Indiana University Press, pp 334-386 